\title{
UNSTABLE AND STABLE GALAXY MODELS
}

\author{
YAN GUO AND ZHIWU LIN
}

\begin{abstract}
To determine the stability and instability of a given steady galaxy configuration is one of the fundamental problems in the Vlasov theory for galaxy dynamics. In this article, we study the stability of isotropic spherical symmetric galaxy models $f_{0}(E)$, for which the distribution function $f_{0}$ depends on the particle energy $E$ only. In the first part of the article, we derive the first sufficient criterion for linear instability of $f_{0}(E): f_{0}(E)$ is linearly unstable if the second-order operator

$$
A_{0} \equiv-\Delta+4 \pi \int f_{0}^{\prime}(E)\{I-\mathcal{P}\} d v
$$

has a negative direction, where $\mathcal{P}$ is the projection onto the function space $\{g(E, L)\}, L$ being the angular momentum [see the explicit formulae (27) and [26] ]. In the second part of the article, we prove that for the important King model, the corresponding $A_{0}$ is positive definite. Such a positivity leads to the nonlinear stability of the King model under all spherically symmetric perturbations.
\end{abstract}

\section{INTRODUCTION}

A galaxy is an ensemble of billions of stars, which interact by the gravitational field which they create collectively. For galaxies, the collisional relaxation time is much longer than the age of the universe ([8]). The collisions can therefore be ignored and the galactic dynamics is well described by the Vlasov - Poisson system (collisionless Boltzmann equation)

$$
\partial_{t} f+v \cdot \nabla_{x} f-\nabla_{x} U \cdot \nabla_{v} f=0, \quad \Delta U=4 \pi \int_{\mathbf{R}^{3}} f(t, x, v) d v,
$$

where $(x, v) \in \mathbb{R}^{3} \times \mathbb{R}^{3}, f(t, x, v)$ is the distribution function and $U_{f}(t, x)$ is its gravitational potential. The Vlasov-Poisson system can also be used to describe the dynamics of globular clusters over their period of orbital revolutions (11]). One of the central questions in such galactic problems, which has attracted considerable attention in the astrophysics literature, of [7, 8], 11, [31] and the references there, is to determine dynamical stability of steady galaxy models. Stability study can be used to test a proposed configuration as a model for a real stellar system. On the other hand, instabilities of steady galaxy models can be used to explain some of the striking irregularities of galaxies, such as spiral arms as arising from the instability of an initially featureless galaxy disk ([7]), ([32]).

In this article, we consider stability of spherical galaxies, which are the simplest elliptical galaxy models. Though most elliptical galaxies are known to be nonspherical, the study of instability and dynamical evolution of spherical galaxies could be useful to understand more complicated and practical galaxy models . By Jeans's Theorem, a steady spherical galaxy is of the form

$$
f_{0}(x, v) \equiv f_{0}\left(E, L^{2}\right),
$$


where the particle energy and total momentum are

$$
E=\frac{1}{2}|v|^{2}+U_{0}(x), L^{2}=|x \times v|^{2},
$$

and $U_{0}(x)=U_{0}(|x|)$ satisfies the self-consistent Poisson equation. The isotropic models take the form

$$
f_{0}(x, v) \equiv f_{0}(E) .
$$

The cases when $f_{0}^{\prime}(E)<0$ has been widely studied and these models are known to be linearly stable to both radial ([9]) and non-radial perturbations ([2]). The well-known Casimir-Energy functional (as a Liapunov functional)

$$
\mathcal{H}(f) \equiv \iint Q(f)+\frac{1}{2} \iint|v|^{2} f-\frac{1}{8 \pi} \int\left|\nabla_{x} U_{f}\right|^{2},
$$

is constant along the time evolution. If $f_{0}^{\prime}(E)<0$, we can choose the Casimir function $Q_{0}$ such that

$$
Q_{0}^{\prime}\left(f_{0}(E)\right) \equiv-E
$$

for all $E$. By a Taylor expansion of $\mathcal{H}(f)-\mathcal{H}\left(f_{0}\right)$, it follows that formally the first variation at $f_{0}$ is zero, that is, $\mathcal{H}^{(1)}\left(f_{0}(E)\right)=0$ (on the support of $f_{0}(E)$ ), and the second order variation of $\mathcal{H}$ at $f_{0}$ is

$$
\mathcal{H}_{f_{0}}^{(2)}[g] \equiv \frac{1}{2} \iint_{\left\{f_{0}>0\right\}} \frac{g^{2}}{-f_{0}^{\prime}(E)} d x d v-\frac{1}{8 \pi} \int\left|\nabla_{x} U_{g}\right|^{2} d x
$$

where $Q^{\prime \prime}\left(f_{0}\right)=\frac{1}{-f_{0}^{\prime}(E)}, g=f-f_{0}$ and $\Delta U_{g}=\int g d v$. In the 1960s, Antonov ([1], 2]) proved that

$$
\mathcal{H}_{f_{0}}^{(2)}[D h]=\iint \frac{|D h|^{2}}{\left|f_{0}^{\prime}(E)\right|} d x d v-\frac{1}{4 \pi} \int\left|\nabla \psi_{h}\right|^{2} d x
$$

is positive definite for a large class of monotone models. Here

$$
D=v \cdot \nabla_{x}-\nabla_{x} U_{0} \cdot \nabla_{v},
$$

$h(x, v)$ is odd in $v$ and $-\Delta \psi=\int D h d v$. He showed that such a positivity is equivalent to the linear stability of $f_{0}(E)$. In [9, Doremus, Baumann and Feix proved the radial stability of any monotone spherical models. Their proof was further clarified and simplified in [10], 37], 22], and more recently in [33], 21]. In particular, this implies that any monotone isotropic models are at least linearly stable.

Unfortunately, despite its importance and a lot of research (e.g., [20, [5], 6], [13]), to our knowledge, no rigorous and explicit instability criterion of non-monotone models has been derived. When $f_{0}^{\prime}(E)$ changes sign, functional $\mathcal{H}_{f_{0}}^{(2)}$ is indefinite and it gives no stability information, although it seems to suggest that these models are not energy minimizers under symplectic perturbations. In this paper, we first obtain the following instability criterion for general spherical galaxies. For any function $g$ with compact support within the support of $f_{0}(E)$, we define the $\left|f_{0}^{\prime}(E)\right|$-weighted $L^{2}\left(\mathbf{R}^{3} \times \mathbf{R}^{3}\right)$ space $L_{\left|f_{0}^{\prime}\right|}^{2}$ with the norm $\|\cdot\|_{\left|f_{0}^{\prime}\right|}$ as

$$
\|h\|_{\left|f_{0}^{\prime}\right|}^{2} \equiv \iint\left|f_{0}^{\prime}(E)\right| h^{2} d x d v .
$$


Theorem 1.1. Assume that $f_{0}(E)$ has a compact support in $x$ and $v$, and $f_{0}^{\prime}$ is bounded. For $\phi \in H^{1}$, define the quadratic form

$$
\left(A_{0} \phi, \phi\right)=\int|\nabla \phi|^{2} d x+4 \pi \iint f_{0}^{\prime}(E)(\phi-\mathcal{P} \phi)^{2} d x d v,
$$

where $\mathcal{P}$ is the projector of $L_{\left|f_{0}^{\prime}\right|}^{2}$ to

$$
\text { ker } D=\left\{g\left(E, L^{2}\right)\right\} \text {, }
$$

and more explicitly $\mathcal{P} \phi$ is given by (18) for radial functions and (26) for general functions. If there exists $\phi_{0} \in H^{1}$ such that

$$
\left(A_{0} \phi_{0}, \phi_{0}\right)<0
$$

then there exists $\lambda_{0}>0$ and $\phi \in H^{2}, f(x, v)$ given by (14), such that $e^{\lambda_{0} t}[f, \phi]$ is a growing mode to the Vlasov-Poisson system (11) linearized around $\left[f_{0}(E), U_{f_{0}}\right]$.

A similar instability criterion can be obtained for symmetry preserving perturbations of anisotropic spherical models $f_{0}\left(E, L^{2}\right)$, see Remark 2. We note that the term $\mathcal{P} \phi$ in the instability criterion is highly non-local and this reflects the collective nature of stellar instability. The proof of Theorem 1.1 is by extending an approach developed in 25] for 1D Vlasov-Poisson, which has recently been generalized to Vlasov-Maxwell systems ([26], [28]). There are two elements in this approach. One is to formulate a family of dispersion operators $A_{\lambda}$ for the potential, depending on a positive parameter $\lambda$. The existence of a purely growing mode is reduced to find a parameter $\lambda_{0}$ such that the $A_{\lambda_{0}}$ has a kernel. The key observation is that these dispersion operators are self-adjoint due to the reversibility of the particle trajectories. Then a continuation argument is applied to find the parameter $\lambda_{0}$ corresponding to a growing mode, by comparing the spectra of $A_{\lambda}$ for very small and large values of $\lambda$. There are two new complications in the stellar case. First, the essential spectrum of $A_{\lambda}$ is $[0,+\infty)$ and thus we need to make sure that the continuation does not end in the essential spectrum. This is achieved by using some compactness property due to the compact support of the stellar model. Secondly, it is more tricky to find the limit of $A_{\lambda}$ when $\lambda$ tends to zero. For that, we need an ergodic lemma (Lemma 2.4) and use the integrable nature of the particle dynamics in a central field to derive an expression for the projection $\mathcal{P} \phi$ appeared in the limit.

In the second part of the article, we further study the nonlinear (dynamical) stability of the normalized King model:

$$
f_{0}=\left[e^{E_{0}-E}-1\right]_{+}
$$

motivated by the study of the operator $A_{0}$. The famous King model describes isothermal galaxies and the core of most globular clusters 24]. Such a model provides a canonical form for many galaxy models widely used in astronomy. Even though $f_{0}^{\prime}<0$ for the King model, it is important to realize that, because of the Hamiltonian nature of the Vlasov-Poisson system (1), linear stability fails to imply nonlinear stability (even in the finite dimensional case). The Liapunov functional is usually required to prove nonlinear stability. In the Casimir-energy functional (2), it is natural to expect that the positivity of such a quadratic form $\mathcal{H}_{f_{0}}^{(2)}[g]$ should imply stability for $f_{0}(E)$. However, there are at least two serious mathematical difficulties. First of all, it is very challenging to use the positivity of $\mathcal{H}_{f_{0}}^{(2)}[g]$ to control higher 
order remainder in $\mathcal{H}(f)-\mathcal{H}\left(f_{0}\right)$ to conclude stability [38. For example, one of the remainder terms is $f^{3}$ whose $L^{2}$ norm is difficult to be bounded by a power of the stability norm. The non-smooth nature of $f_{0}(E)$ also causes trouble here. Second of all, even if one can succeed in controlling the nonlinearity, the positivity of $H_{f_{0}}^{(2)}[g]$ is only valid for certain perturbation of the form $g=D h$ 22. It is not clear at all if any arbitrary, general perturbation can be reduced to the form $D h$. To overcome these two difficulties, a direct variational approach was initiated by Wolansky [39, then further developed systematically by Guo and Rein in [14, [15], 117, [18, [19]. Their method avoids entirely the delicate analysis of the second order variation $\mathcal{H}_{f_{0}}^{(2)}$ in (3), which has led to first rigorous nonlinear stability proof for a large class of $f_{0}(E)$. The high point of such a program is the nonlinear stability proof for every polytrope [18 $f_{0}(E)=\left(E_{0}-E\right)_{+}^{k}$. Their basic idea is to construct galaxy models by solving a variational problem of minimizing the energy under some constraints of Casimir invariants. A concentration-compactness argument is used to show the convergence of the minimizing sequence. All the models constructed in this way are automatically stable.

Unfortunately, despite its success, the King model can not be studied by such a variational approach. The Casimir function for a normalized King model is

$$
Q_{0}(f)=(1+f) \ln (1+f)-1-f,
$$

which has very slow growth for $f \rightarrow \infty$. As a result, the direct variational method fails. Recently, Guo and Rein [21] proved nonlinear radial stability among a class of measure-preserving perturbations

$$
\mathcal{S}_{f_{0}} \equiv\left[f\left(t, r, v_{r}, L\right) \geq 0: \int Q(f, L)=\int Q\left(f_{0}, L\right) \text {, for } Q \in C_{c}^{\infty} \text { and } Q(0, L) \equiv 0 .\right] \text {. }
$$

The basic idea is to observe that for perturbations in the class $\mathcal{S}_{f_{0}}$, one can write $g=f-f_{0}$ as $D h=\{h, E\}$. Therefore, $\mathcal{H}_{f_{0}}^{(2)}[g]=\mathcal{H}_{f_{0}}^{(2)}[D h]$, for which the positivity was proved in 22 for radial perturbations. To avoid the difficulty of controlling the remainder term by $\mathcal{H}_{f_{0}}^{(2)}[g]$, an indirect contradiction argument was used in [21.

As our second main result of this article, we establish nonlinear stability of King's model for general perturbations with spherical symmetry:

Theorem 1.2. The King's model $f_{0}=\left[e^{E_{0}-E}-1\right]_{+}$is nonlinearly stable under spherically symmetric perturbations in the following sense: given any $\varepsilon>0$ there exists $\varepsilon_{1}>0$ such that for any compact supported initial data $f(0) \in C_{c}^{1}$ with spherical symmetry, if $d\left(f(0), f_{0}\right)<\varepsilon_{1}$ then

$$
\sup _{0 \leq t<\infty} d\left(f(t), f_{0}\right)<\varepsilon,
$$

where the distance functional $d\left(f, f_{0}\right)$ is defined by (35).

For the proof, we extended the approach in [27] for the $1 \frac{1}{2} D$ Vlasov-Maxwell model. To prove nonlinear stability, we study the Taylor expansion of $\mathcal{H}(f)-\mathcal{H}\left(f_{0}\right)$. Two difficulties as mentioned before are: to prove the positivity of the quadratic form and to control the remainder. We use two ideas introduced in 27. The first idea is to use any finite number of Casimir functional $Q_{i}\left(f, L^{2}\right)$ as constraints. The difference from 21 is that we do not impose $Q_{i}\left(f, L^{2}\right)=Q_{i}\left(f_{0}, L^{2}\right)$ in the perturbation class, but expand the invariance equation $Q_{i}\left(f(t), L^{2}\right)-Q_{i}\left(f_{0}, L^{2}\right)=$ 
$Q_{i}\left(f(0), L^{2}\right)-Q_{i}\left(f_{0}, L^{2}\right)$ to the first order. In this way, we get a constraint for $g=f-f_{0}$ in the form that the coefficient of its projection to $\partial_{1} Q_{i}\left(f_{0}, L^{2}\right)$ is small. Putting these constraints together, we deduce that a finite dimensional projection of $g$ to the space spanned by $\left\{\partial_{1} Q_{i}\left(f_{0}, L^{2}\right)\right\}$ is small. To control the remainder term, we use a duality argument. Noting that it is much easier to control the potential $\phi$, we use a Legendre transformation to reduce the nonlinear term in $g$ to a new one in $\phi$ only. The key observation is that the constraints on $g$ in the projection form are nicely suited to the Legendre transformation and yields a non-local nonlinear term in $\phi$ only with the projections kept. By performing a Taylor expansion of this non-local nonlinear term in $\phi$, the quadratic form becomes a truncated version of $\left(A_{0} \phi, \phi\right)$ defined by (6) , whose positivity can be shown to be equivalent to that of Antonov functional. The the remainder term now is only in terms of $\phi$ and can be easily controlled by the quadratic form. The new complication in the stellar case is that the steady distribution $f_{0}(E)$ is non-smooth and compactly supported. Therefore, we split the perturbation $g$ into inner and outer parts, according to the support of $f_{0}$. For the inner part, we use the above constrainted duality argument and the outer part is estimated separately.

\section{An Instability Criterion}

We consider a steady distribution

$$
f_{0}(x, v)=f_{0}(E)
$$

has a bounded support in $x$ and $v$ and $f_{0}^{\prime}$ is bounded, where the particle energy $E=\frac{1}{2}|v|^{2}+U_{0}(x)$. The steady gravitational potential $U_{0}(x)$ satisfies a nonlinear Poisson equation

$$
\Delta U_{0}=4 \pi \int f_{0} d v
$$

The linearized Vlasov-Poisson system is

$$
\partial_{t} f+v \cdot \nabla_{x} f-\nabla_{x} U_{0} \cdot \nabla_{v} f=\nabla_{x} \phi \cdot \nabla_{v} f_{0}, \quad \Delta \phi=4 \pi \int f(t, x, v) d v .
$$

A growing mode solution $\left(e^{\lambda t} f(x, v), e^{\lambda t} \phi(x)\right)$ to (11) with $\lambda>0$ satisfies

$$
\lambda f+v \cdot \nabla_{x} f-\nabla_{x} U_{0} \cdot \nabla_{v} f=f_{0}^{\prime} v \cdot \nabla_{x} \phi .
$$

We define $[X(s ; x, v), V(s ; x, v)]$ as the trajectory of

$$
\left\{\begin{array}{c}
\frac{d X(s ; x, v)}{d s}=V(s ; x, v) \\
\frac{d V(s ; x, v)}{d s}=-\nabla_{x} U_{0}
\end{array}\right.
$$

such that $X(0 ; x, v)=x$, and $V(0 ; x, v)=v$. Notice that the particle energy $E$ is constant along the trajectory. Integrating along such a trajectory for $-\infty \leq s \leq 0$, we have

$$
\begin{aligned}
f(x, v) & =\int_{-\infty}^{0} e^{\lambda s} f_{0}^{\prime}(E) V(s ; x, v) \cdot \nabla_{x} \phi(X(s ; x, v)) d s \\
& =f_{0}^{\prime}(E) \phi(x)-f_{0}^{\prime}(E) \int_{-\infty}^{0} \lambda e^{\lambda s} \phi(X(s ; x, v)) d s .
\end{aligned}
$$


Plugging it back into the Poisson equation, we obtain an equation for $\phi$

$$
-\Delta \phi+\left[4 \pi \int f_{0}^{\prime}(E) d v\right] \phi-4 \pi \int f_{0}^{\prime}(E) \int_{-\infty}^{0} \lambda e^{\lambda s} \phi(X(s ; x, v)) d s d v=0 .
$$

We therefore define the operator $A_{\lambda}$ as

$$
A_{\lambda} \phi \equiv-\Delta \phi+\left[4 \pi \int f_{0}^{\prime}(E) d v\right] \phi-4 \pi \int f_{0}^{\prime}(E) \int_{-\infty}^{0} \lambda e^{\lambda s} \phi(X(s ; x, v)) d s d v .
$$

Lemma 2.1. Assume that $f_{0}(E)$ has a bounded support in $x$ and $v$ and $f_{0}^{\prime}$ is bounded. For any $\lambda>0$, the operator $A_{\lambda}: H^{2} \rightarrow L^{2}$ is self-adjoint with the essential spectrum $[0,+\infty)$.

Proof. We denote

$$
K_{\lambda} \phi=-4 \pi\left[\int f_{0}^{\prime}(E) d v\right] \phi+4 \pi \int f_{0}^{\prime}(E) \int_{-\infty}^{0} \lambda e^{\lambda s} \phi(X(s ; x, v)) d s d v .
$$

Recall that $f_{0}(x, v)=f_{0}(E)$ has a compact support $\subset S \subset \mathbb{R}_{x}^{3} \times \mathbb{R}_{v}^{3}$. We may assume $S=S_{x} \times S_{v}$, both balls in $\mathbb{R}^{3}$. Let $\chi=\chi(|x|)$ be a smooth cut-off function for the spatial support of $f_{0}$ in the physical space $S_{x}$; that is, $\chi \equiv 1$ on the spatial support of $f_{0}$ and has compact support inside $S_{x}$. Let $M_{\chi}$ be the operator of multiplication by $\chi$. Then $K_{\lambda}=K_{\lambda} M_{\chi}=M_{\chi} K_{\lambda}=M_{\chi} K_{\lambda} M_{\chi}$. Indeed,

$$
f_{0}^{\prime}(x, v)=f_{0}^{\prime}(X(s ; x, v), V(s ; x, v))
$$

because of the invariance of $E$ under the flow. So

$$
\begin{aligned}
\left(K_{\lambda} \phi\right)(x) & =-4 \pi\left[\int f_{0}^{\prime}(E) d v\right] \phi+4 \pi \int f_{0}^{\prime}(E) \int_{-\infty}^{0} \lambda e^{\lambda s} \phi(X(s ; x, v)) d s d v \\
& =-4 \pi\left[\int f_{0}^{\prime}(E) d v\right] \phi+4 \pi \iint_{-\infty}^{0} \lambda e^{\lambda s}\left(f_{0}^{\prime}(E) \phi\right)(X(s ; x, v)) d s d v \\
& =\left(M_{\chi} K_{\lambda} M_{\chi} \phi\right)(x) .
\end{aligned}
$$

First we claim that

$$
\left\|K_{\lambda}\right\|_{L^{2} \rightarrow L^{2}} \leq 8 \pi\left|\int\right| f_{0}^{\prime}(E)|d v|_{\infty} .
$$

Indeed, the $L^{2}$ norm for the first term in $K_{\lambda}$ is easily bounded by $4 \pi\left|\int f_{0}^{\prime}(E) d v\right|_{\infty}$. For the second term, we have for any $\psi \in L^{2}$,

$$
\begin{aligned}
& \left|\int_{-\infty}^{0} \iint 4 \pi \lambda e^{\lambda s} f_{0}^{\prime}(E) \phi(X(s ; x, v)) d s d v \psi(x) d x\right| \\
& \leq 4 \pi \int_{-\infty}^{0} \lambda e^{\lambda s}\left(\iint\left|f_{0}^{\prime}(E)\right| \phi^{2}(X(s ; x, v)) d v d x\right)^{\frac{1}{2}}\left(\iint\left|f_{0}^{\prime}(E)\right| \psi^{2}(x) d v d x\right)^{\frac{1}{2}} d s \\
& =4 \pi \int_{-\infty}^{0} \lambda e^{\lambda s}\left(\iint\left|f_{0}^{\prime}(E)\right| \phi^{2}(x) d v d x\right)^{\frac{1}{2}}\left(\iint\left|f_{0}^{\prime}(E)\right| \psi^{2}(x) d v d x\right)^{\frac{1}{2}} d s \\
& =4 \pi\left(\iint\left|f_{0}^{\prime}(E)\right| \phi^{2}(x) d v d x\right)^{\frac{1}{2}}\left(\iint\left|f_{0}^{\prime}(E)\right| \psi^{2}(x) d v d x\right)^{\frac{1}{2}} \\
& \leq 4 \pi\left|\int\right| f_{0}^{\prime}(E)|d v|_{\infty}\|\phi\|_{2}\|\psi\|_{2} .
\end{aligned}
$$


Moreover, we have that $K_{\lambda}$ is symmetric Indeed, for fixed $s$, by making a change of variable $(y, w) \rightarrow(X(s ; x, v), V(s ; x, v))$, so that $(z, v)=(X(-s ; y, w), V(-s ; y, w))$, we deduce that

$$
\begin{aligned}
& \iint 4 \pi f_{0}^{\prime}(E) \int_{-\infty}^{0} \lambda e^{\lambda s} \phi(X(s ; x, v)) d s d v \psi(x) d x \\
& =\int_{-\infty}^{0} \lambda e^{\lambda s} \iint 4 \pi f_{0}^{\prime}(E) \phi(y) \psi(X(-s ; y, w)) d y d w d s \\
& =\iint 4 \pi f_{0}^{\prime}(E) \int_{-\infty}^{0} \lambda e^{\lambda s} \psi(X(-s ; y,-w)) \phi(y) d y d w d s \\
& =\iint 4 \pi f_{0}^{\prime}(E) \int_{-\infty}^{0} \lambda e^{\lambda s} \psi(X(s ; x, v)) \phi(x) d v d x d s .
\end{aligned}
$$

Here we have used the fact $[X(s ; y, w), V(s ; y, w)]=[X(-s ; y,-w),-V(s ; y,-w)]$ in the last line. Hence

$$
\left(K_{\lambda} \phi, \psi\right)=\left(\phi, K_{\lambda} \psi\right) .
$$

Since $K_{\lambda}=K_{\lambda} M_{\chi}$ and $M_{\chi}$ is compact from $H^{2}$ into $L^{2}$ space with support in $S_{x}$, so $K_{\lambda}$ is relatively compact with respect to $-\Delta$. Thus by Kato-Relich and Weyl's Theorems, $A_{\lambda}: H^{2} \rightarrow L^{2}$ is self-adjoint and $\sigma_{\text {ess }}\left(A_{\lambda}\right)=\sigma_{\text {ess }}(-\Delta)$.

Lemma 2.2. Assume that $f_{0}^{\prime}(E)$ has a bounded support in $x$ and $v$ and $f_{0}^{\prime}$ is bounded. Let

$$
k(\lambda)=\inf _{\phi \in D\left(A_{\lambda}\right),\|\phi\|_{2}=1}\left(\phi, A_{\lambda} \phi\right),
$$

then $k(\lambda)$ is a continuous function of $\lambda$ when $\lambda>0$. Moreover, there exists $0<$ $\Lambda<\infty$ such that for $\lambda>\Lambda$

$$
k(\lambda) \geq 0 .
$$

Proof. Fix $\lambda_{0}>0, \phi \in D\left(A_{\lambda}\right)$, and $\|\phi\|_{2}=1$. Then

$$
\begin{aligned}
k\left(\lambda_{0}\right) & \leq\left(\phi, A_{\lambda_{0}} \phi\right) \\
& \leq\left(\phi, A_{\lambda} \phi\right)+\left|\left(\phi, A_{\lambda_{0}} \phi\right)-\left(\phi, A_{\lambda} \phi\right)\right| \\
& \leq\left(\phi, A_{\lambda} \phi\right)+4 \pi \iint\left|f_{0}^{\prime}(E)\right| \int_{-\infty}^{0}\left[\lambda e^{\lambda s}-\lambda_{0} e^{\lambda_{0} s}\right] \phi(X(s ; x, v)) \phi(x) d s d v d x \\
& \leq\left(\phi, A_{\lambda} \phi\right)+4 \pi \iint\left|f_{0}^{\prime}(E)\right| \int_{-\infty}^{0} \int_{\lambda_{0}}^{\lambda}\left[\tilde{\lambda}|s| e^{\tilde{\lambda} s}+e^{\tilde{\lambda} s}\right] d \tilde{\lambda} \phi(X(s ; x, v)) \phi(x) d s d v d x \\
& \leq\left(\phi, A_{\lambda} \phi\right)+C \int_{-\infty}^{0} \int_{\lambda_{0}}^{\lambda}\left[\tilde{\lambda}|s| e^{\tilde{\lambda} s}+e^{\tilde{\lambda} s}\right] d \tilde{\lambda} d s \\
& \leq\left(\phi, A_{\lambda} \phi\right)+C\left|\ln \lambda-\ln \lambda_{0}\right| .
\end{aligned}
$$

We therefore deduce that by taking the infimum over all $\phi$,

$$
k\left(\lambda_{0}\right) \leq k(\lambda)+C\left|\ln \lambda-\ln \lambda_{0}\right| .
$$

Same argument also yields $k(\lambda) \leq k\left(\lambda_{0}\right)+C\left|\ln \lambda-\ln \lambda_{0}\right|$.Thus $\left|k\left(\lambda_{0}\right)-k(\lambda)\right| \leq$ $C\left|\ln \lambda-\ln \lambda_{0}\right|$ and $k(\lambda)$ is continuous for $\lambda>0$.

To prove (17), by (14), we recall from Sobolev's inequality in $\mathbf{R}^{3}$ 


$$
\begin{aligned}
\left|\left(K_{\lambda} \phi, \psi\right)\right| & =\left|\iint 4 \pi f_{0}^{\prime}(E) e^{\lambda s} \nabla \phi(X(s ; x, v)) V(s) d s d v \psi(x) d x\right| \\
& \leq \int_{-\infty}^{0} e^{\lambda s}\left(\iint|\psi|^{2}\left|f_{0}^{\prime}(E)\right| d v d x\right)^{1 / 2} \cdot \\
& \times\left[\iint|\nabla \phi(X(s))|^{2}\left|f_{0}^{\prime}(E) \| V(s)\right|^{2} d x d v\right]^{1 / 2} d s \\
& \left.=\int_{-\infty}^{0} e^{\lambda s}\left(\iint|\psi|^{2}\left|f_{0}^{\prime}(E)\right| d v d x\right)^{1 / 2} \iint v^{2}|\nabla \phi(x)|^{2}\left|f_{0}^{\prime}(E)\right| d x d v\right]^{1 / 2} d s \\
& \leq \frac{C}{\lambda}\|\psi\|_{6}\|\nabla \phi\|_{2} \leq \frac{C}{\lambda}\|\nabla \psi\|_{2}\|\nabla \phi\|_{2},
\end{aligned}
$$

since $f_{0}$ has compact support. Therefore,

$$
\left(A_{\lambda} \phi, \phi\right)=\|\nabla \phi\|^{2}-\left(K_{\lambda} \phi, \phi\right) \geq\left(1-\frac{C}{\lambda}\right)\|\nabla \phi\|^{2} \geq 0
$$

for $\lambda$ large.

We now compute $\lim _{\lambda \rightarrow 0+} A_{\lambda}$. We first consider the case when the test function $\phi$ is spherically symmetric.

Lemma 2.3. For spherically symmetric function $\phi(x)=\phi(|x|)$, we have

$$
\begin{aligned}
\lim _{\lambda \rightarrow 0+}\left(A_{\lambda} \phi, \phi\right)=\left(A_{0} \phi, \phi\right) \equiv \int|\nabla \phi|^{2} d x+4 \pi \iint f_{0}^{\prime}(E) d v \phi^{2} d x \\
\quad-32 \pi^{3} \int_{\min U_{0}}^{E} \int_{0}^{\infty} f_{0}^{\prime}(E) \frac{\left(\int_{r_{1}(E, L)}^{r_{2}(E, L)} \frac{\phi d r}{\sqrt{2\left(E-U_{0}-L^{2} / 2 r^{2}\right)}}\right)^{2}}{\int_{r_{1}(E, L)}^{r_{2}(E, L)} \frac{d r}{\sqrt{2\left(E-U_{0}-L^{2} / 2 r^{2}\right)}}} d L d E \\
=\int|\nabla \phi|^{2}+32 \pi^{3} \int f_{0}^{\prime}(E) \int_{r_{1}(E, L)}^{r_{2}(E, L)}(\phi-\bar{\phi})^{2} \frac{d r d E d L}{\sqrt{2\left(E-U_{0}-L^{2} / 2 r^{2}\right)}} .
\end{aligned}
$$

Proof. Given the steady state $f_{0}(E), U_{0}(|x|)$ and any radial function $\phi(|x|)$. To find the limit of

$$
\begin{aligned}
\left(A_{\lambda} \phi, \phi\right) & =\int|\nabla \phi|^{2} d x+4 \pi \iint f_{0}^{\prime}(E) d v \phi^{2} d x \\
& -4 \pi \iint f_{0}^{\prime}(E)\left(\int_{-\infty}^{0} \lambda e^{\lambda s} \phi(X(s ; x, v)) d s\right) \phi(x) d x d v
\end{aligned}
$$

we study the following

$$
\lim _{\lambda \rightarrow 0+} \int_{-\infty}^{0} \lambda e^{\lambda s} \phi(X(s ; x, v)) d s .
$$

Note that we only need to study (20) for points $(x, v)$ with $E=\frac{1}{2}|v|^{2}+U_{0} \mid(x \mid)<E_{0}$ and $L=|x \times v|>0$, because in the third integral of (19) $f_{0}^{\prime}(E)$ has support in 
$\left\{E<E_{0}\right\}$ and the set $\{L=0\}$ has a zero measure. We recall the linearized VlasovPoisson system in the $r, v_{r}, L$ coordinates takes the form

$$
\begin{aligned}
\partial_{t} f+v_{r} \partial_{r} f+\left(\frac{L}{r^{3}}-\partial_{r} U_{0}\right) \partial_{v_{r}} f & =\partial_{r} U_{f} \partial_{v_{r}} f_{0} \\
\partial_{r r} U_{f}+\frac{2}{r} \partial_{r} U_{f} & =4 \pi \int f d v
\end{aligned}
$$

For the corresponding linearized system, for points $(x, v)$ with $E<E_{0}$ and $L>0$, the trajectory of $(X(s ; x, v), V(s ; x, v))$ in the coordinate $(r, E, L)$ is a periodic motion described by the ODE (see [8])

$$
\begin{aligned}
\frac{d r(s)}{d s} & =v_{r}(s), \\
\frac{d v_{r}(s)}{d s} & =-U_{0}^{\prime}(r)+\frac{L^{2}}{r^{3}}
\end{aligned}
$$

with the period

$$
T(E, L)=2 \int_{r_{1}(E, L)}^{r_{2}(E, L)} \frac{d r}{\sqrt{2\left(E-U_{0}-L^{2} / 2 r^{2}\right)}},
$$

where $0<r_{1}(E, L) \leq r_{2}(E, L)<+\infty$ are zeros of $E-U_{0}-L^{2} / 2 r^{2}$.So by Lin's lemma in [[25],

$$
\lim _{\lambda \rightarrow 0} \int_{-\infty}^{0} \lambda e^{\lambda s} \phi(X(s ; x, v)) d s=\frac{1}{T} \int_{0}^{T} \phi(X(s ; x, v)) d s .
$$

Since $\phi(X(s ; x, v)=\phi(r(s))$, a change of variable from $s \rightarrow r(s)$ leads to

$$
\int_{0}^{T} \phi(X(s ; x, v)) d s=2 \int_{r_{1}}^{r_{2}} \frac{\phi(r) d r}{\sqrt{2\left(E-U_{0}-L^{2} / 2 r^{2}\right)}} .
$$

For any function $g(r, E, L)$, we define its trajectory average as

$$
\bar{g}(E, L) \equiv \frac{\int_{r_{1}(E, L)}^{r_{2}(E, L)} \frac{g(r, E, L) d r}{\sqrt{2\left(E-U_{0}-L^{2} / 2 r^{2}\right)}}}{\int_{r_{1}(E, L)}^{r_{2}(E, L)} \frac{d r}{\sqrt{2\left(E-U_{0}-L^{2} / 2 r^{2}\right)}}} .
$$

Then

$$
\lim _{\lambda \rightarrow 0+} \int_{-\infty}^{0} \lambda e^{\lambda s} \phi(X(s ; x, v)) d s=2 \int_{r_{1}}^{r_{2}} \frac{\phi(r) d r}{\sqrt{2\left(E-U_{0}-L^{2} / 2 r^{2}\right)}} / T(E, L)=\bar{\phi}(E, L)
$$


and the integrand in third term of (19) converges pointwise to $f_{0}^{\prime}(E) \bar{\phi} \phi$. Thus by the dominated convergence theorem, we have

$$
\begin{aligned}
\lim _{\lambda \rightarrow 0+}\left(A_{\lambda} \phi, \phi\right)= & \int|\nabla \phi|^{2} d x+4 \pi \iint f_{0}^{\prime}(E) \phi^{2} d x d v-4 \pi \iint f_{0}^{\prime}(E) \bar{\phi} \phi d x d v \\
= & \int|\nabla \phi|^{2} d x+4 \pi \iint f_{0}^{\prime}(E) \phi^{2} d x d v \\
& \quad-32 \pi^{3} \int_{\min U_{0}}^{E} \int_{0}^{\infty} f_{0}^{\prime}(E) \int_{r_{1}(E, L)}^{r_{2}(E, L)} \bar{\phi}(E, L) \phi(r) \frac{d r d E d L}{\sqrt{2\left(E-U_{0}-L / 2 r^{2}\right)}} \\
= & \int|\nabla \phi|^{2} d x+4 \pi \iint f_{0}^{\prime}(E) \phi^{2} d x d v \\
& \quad-32 \pi^{3} \int_{\min U_{0}}^{E} \int_{0}^{\infty} f_{0}^{\prime}(E) \frac{\left(\int_{r_{1}(E, L)}^{r_{2}(E, L)} \frac{\phi d r}{\sqrt{2\left(E-U_{0}-L / 2 r^{2}\right)}}\right)^{2}}{\int_{r_{1}(E, L)}^{r_{2}(E, L)} \frac{d r}{\sqrt{2\left(E-U_{0}-L / 2 r^{2}\right)}}} d E d L \\
= & \int|\nabla \phi|^{2}+32 \pi^{3} \int f_{0}^{\prime}(E) \int_{r_{1}(E, L)}^{r_{2}(E, L)}(\phi-\bar{\phi})^{2} \frac{d r d E d L}{\sqrt{2\left(E-U_{0}-L / 2 r^{2}\right)}} .
\end{aligned}
$$

This finishes the proof of the lemma.

To compute $\lim _{\lambda \rightarrow 0+}\left(A_{\lambda} \phi, \phi\right)$ for more general test function $\phi$, we use the following ergodic lemma which is a direct generalization of the result in [26].

Lemma 2.4. Consider the solution $(P(s ; p, q), Q(s ; p, q))$ to be the solution of a Hamiltonian system

$$
\begin{aligned}
& \dot{P}=\partial_{q} H(P, Q) \\
& \dot{Q}=-\partial_{p} H(P, Q)
\end{aligned}
$$

with $(P(0), Q(0))=(p, q) \in \mathbf{R}^{n} \times \mathbf{R}^{n}$. Denote

$$
\mathcal{Q}^{\lambda} m=\int_{-\infty}^{0} \lambda e^{\lambda s} m(P(s), Q(s)) d s .
$$

Then for any $m(p, q) \in L^{2}\left(\mathbf{R}^{n} \times \mathbf{R}^{n}\right)$, we have $\mathcal{Q}^{\lambda} m \rightarrow \mathcal{P} m$ strongly in $L^{2}\left(\mathbf{R}^{n} \times \mathbf{R}^{n}\right)$. Here $\mathcal{P}$ is the projection operator of $L^{2}\left(\mathbf{R}^{n} \times \mathbf{R}^{n}\right)$ to the kernel of the transport operator $D=\partial_{q} H \partial_{p}-\partial_{p} H \partial_{q}$ and $\mathcal{P} m$ is the phase space average of $m$ in the set traced by the trajectory.

Proof. Denote $U(s): L^{2}\left(\mathbf{R}^{n} \times \mathbf{R}^{n}\right) \rightarrow L^{2}\left(\mathbf{R}^{n} \times \mathbf{R}^{n}\right)$ to be the unitary semigroup $U(s) m=m(P(s), Q(s))$. By Stone Theorem (40]), $U(s)$ is generated by $i R=D$, where $R=-i D$ is self-adjoint and

$$
U(s)=\int_{-\infty}^{+\infty} e^{i \alpha s} d M_{\alpha}
$$

where $\left\{M_{\alpha} ; \alpha \in \mathbf{R}^{1}\right\}$ is spectral measure of $R$. So

$$
\int_{-\infty}^{0} \lambda e^{\lambda s} m(P(s), Q(s)) d s=\int_{-\infty}^{0} \lambda e^{\lambda s} \int_{\mathbb{R}} e^{i \alpha s} d M_{\alpha} m d s=\int_{\mathbb{R}} \frac{\lambda}{\lambda+i \alpha} d M_{\alpha} m .
$$


On the other hand, the projection is $\mathcal{P}=M_{\{0\}}=\int_{\mathbb{R}} \xi d M_{\alpha}$ where $\xi(\alpha)=0$ for $\alpha \neq 0$ and $\xi(0)=1$. Therefore

$$
\left\|\int_{-\infty}^{0} \lambda e^{\lambda s} m(P(s), Q(s)) d s-\mathcal{P} m\right\|_{\mathbf{L}^{2}}^{2}=\int_{\mathbb{R}}\left|\frac{\lambda}{\lambda+i \alpha}-\xi(\alpha)\right|^{2} d\left\|M_{\alpha} m\right\|_{\mathbf{L}^{2}}^{2}
$$

by orthogonality of the spectral projections. By the dominated convergence theorem this expression tends to 0 as $\lambda \rightarrow 0+$, as we wished to prove. The explaination of $\mathcal{P} m$ as the phase space average of $m$ is in our remark below.

Remark 1. Since $\int_{-\infty}^{0} \lambda e^{\lambda s} d s=1$, the function

$$
\left(\mathcal{Q}^{\lambda} m\right)(x, v)=\int_{-\infty}^{0} \lambda e^{\lambda s} m(P(s), Q(s)) d s
$$

is a weighted time average of the observable $m$ along the particle trajectory. By the same proof of Lemma 2.4, we have

$$
\lim _{T \rightarrow \infty} \frac{1}{T} \int_{0}^{T} m(P(s), Q(s)) d s=\mathcal{P} m .
$$

But from the standard ergodic theory (3) of Hamiltonian systems, the limit of the above time average in (22) equals the phase space average of $m$ in the set traced by the trajectory. Thus $\mathcal{P} m$ has the meaning of the phase space average of $m$ and Lemma 2.4 states that the limit of the weighted time average (21) yields the same phase space average. In particular, if the particle motion is ergodic in the invariant set $S_{I}$ determined by the invariants $E_{1}, \cdots, I_{k}$, and if $d \sigma_{I}$ denotes the induced measure of $\mathbf{R}^{n} \times \mathbf{R}^{n}$ on $S_{I}$, then

$$
\mathcal{P} m=\frac{1}{\sigma_{I}\left(S_{I}\right)} \int_{S_{I}} m(p, q) d \sigma_{I}(p, q) .
$$

For integral systems, using action angle variables $\left(J_{1}, \cdots, J_{n} ; \varphi_{1}, \cdots, \varphi_{n}\right)$ we have

$$
(\mathcal{P} m)\left(J_{1}, \cdots, J_{n}\right)=(2 \pi)^{-n} \int_{0}^{2 \pi} \cdots \int_{0}^{2 \pi} m\left(J_{1}, \cdots, J_{n}, \varphi_{1}, \cdots, \varphi_{n}\right) d \varphi_{1}, \cdots d \varphi_{n}
$$

for the generic case with independent frequencies (see [4]).

Recall the weighted $L^{2}$ space $L_{\left|f_{0}^{\prime}\right|}^{2}$ in (5). Then $U(s): L_{\left|f_{0}^{\prime}\right|}^{2} \rightarrow L_{\left|f_{0}^{\prime}\right|}^{2}$ defined by $U(s) m=m(X(s ; x, v), V(s ; x, v))$ is an unitary group, where $(X(s ; x, v), V(s ; x, v))$ is the particle trajectory (13). The generator of $U(s)$ is $D=v \cdot \partial_{x}-\nabla_{x} U_{0} \cdot \nabla_{v}$ and $R=-i D$ is self-adjoint by Stone Theorem. By the same proof, Lemma 2.4 is still valid in $L_{\left|f_{0}^{\prime}\right|}^{2}$. In particular, for any $\phi(x) \in L^{2}\left(\mathbf{R}^{3}\right)$ we have

$$
\int_{-\infty}^{0} \lambda e^{\lambda s} \phi(X(s ; x, v)) d s \rightarrow \mathcal{P} \phi
$$

in $L_{\left|f_{0}^{\prime}\right|}^{2}$, where $\mathcal{P}$ is the projector of $L_{\left|f_{0}^{\prime}\right|}^{2}$ to $\operatorname{ker} D$.

Now we derive an explicit formula for the above limit $\mathcal{P} \phi$. Note that as in the proof of lemma 2.3, we only need to derive the formula of $\mathcal{P} \phi$ for points $(x, v)$ with $E<E_{0}$ and $L>0$. Since $U_{0}(x)=U_{0}(r)$, the particle motion (13) in such a center field is integrable and has been well studied (see e.g. 8, 4). For particles with 
energy $E<E_{0}<0, L>0$ and momentum $\vec{L}=x \times v$, the particle orbit is a rosette in the annulus

$$
A_{E, L}=\left\{r_{1}(E, L) \leq r \leq r_{2}(E, L)\right\}=\left\{E-U_{0}-L^{2} / 2 r^{2} \geq 0\right\},
$$

lying on the orbital plane perpendicular to $\vec{L}$. So we can consider the particle motion to be planar. For such case, the action-angle variables are as follows (see e.g. 30]): the actions variables are

where

$$
J_{r}=\frac{2 \pi}{T(E, L)}, \quad J_{\theta}=L
$$

$$
T(E, L)=2 \int_{r_{1}(E, L)}^{r_{2}(E, L)} \frac{d r}{\sqrt{2\left(E-U_{0}-L^{2} / 2 r^{2}\right)}} .
$$

is the radial period, the angle variable $\varphi_{r}$ is determined by

$$
d \varphi_{r}=\frac{2 \pi}{T(E, L)} \frac{d r}{\sqrt{2\left(E-U_{0}-L^{2} / 2 r^{2}\right)}}
$$

and $\varphi_{\theta}=\theta-\Delta \theta$ where

$$
d(\Delta \theta)=\frac{L r^{-2}-\Omega_{\theta}}{\sqrt{2\left(E-U_{0}-L^{2} / 2 r^{2}\right)}} d r
$$

and

$$
\Omega_{\theta}(E, L)=\frac{1}{T(E, L)} \int_{r_{1}(E, L)}^{r_{2}(E, L)} \frac{L}{r^{2} \sqrt{2\left(E-U_{0}-L^{2} / 2 r^{2}\right)}} d r
$$

is the average angular velocity. For any function $\phi(x) \in H^{2}\left(\mathbf{R}^{3}\right)$, we denote $\phi_{\vec{L}}(r, \theta)$ to be the restriction of $\phi$ in the orbital plane perpendicular to $\vec{L}$. Then by $(24)$, for the generic case when the radial and angular frequencies are independent, we have

$$
\begin{aligned}
(\mathcal{P} \phi)(E, \vec{L}) & =(2 \pi)^{-2} \int_{0}^{2 \pi} \int_{0}^{2 \pi} \phi_{\vec{L}} d \varphi_{\theta} d \varphi_{r} \\
& =\frac{1}{\pi T(E, L)} \int_{r_{1}(E, L)}^{r_{2}(E, L)} \int_{0}^{2 \pi} \frac{\phi_{\vec{L}}(r, \theta) d \theta d r}{\sqrt{2\left(E-U_{0}-L^{2} / 2 r^{2}\right)}} .
\end{aligned}
$$

In particular, for a spherically symmetric function $\phi=\phi(r)$, we recover

$$
(\mathcal{P} \phi)(E, L)=\frac{2}{T(E, L)} \int_{r_{1}(E, L)}^{r_{2}(E, L)} \frac{\phi(r) d r}{\sqrt{2\left(E-U_{0}-L^{2} / 2 r^{2}\right)}} .
$$

We thus conclude the following

Lemma 2.5. Assume that $f_{0}(E)$ has a bounded support in $x$ and $v$ and $f_{0}^{\prime}$ is bounded. For any $\phi \in H^{1}\left(\mathbf{R}^{3}\right)$, we have

$$
\begin{aligned}
\lim _{\lambda \rightarrow 0+}\left(A_{\lambda} \phi, \phi\right) & =\left(A_{0} \phi, \phi\right) \\
& =\int|\nabla \phi|^{2} d x+4 \pi \iint f_{0}^{\prime}(E) d v \phi^{2} d x-4 \pi \iint f_{0}^{\prime}(E)(\mathcal{P} \phi)^{2} d x d v \\
& =\int|\nabla \phi|^{2} d x+4 \pi \iint f_{0}^{\prime}(E)(\phi-\mathcal{P} \phi)^{2} d x d v
\end{aligned}
$$


where $\mathcal{P}$ is the projector of $L_{\left|f_{0}^{\prime}\right|}^{2}$ to $\operatorname{ker} D$ and more explicitly $\mathcal{P} \phi$ is given by (26). The limiting operator $A_{0}$ is

$$
A_{0} \phi=-\Delta \phi+\left[4 \pi \int f_{0}^{\prime}(E) d v\right] \phi-4 \pi \int f_{0}^{\prime}(E) \mathcal{P} \phi d v .
$$

Now we give the proof of the instability criterion.

Proof of Theorem 1.1. We define

$$
\lambda_{*}=\sup _{k(\lambda)<0} \lambda .
$$

By Lemmas 2.1 and 2.5, we deduce that

$$
-\infty<\lambda_{*} \leq \Lambda<\infty .
$$

Therefore, by the continuity of $k(\lambda)$, we have

$$
k\left(\lambda_{*}\right)=0 .
$$

Hence, there exists an increasing sequence of $\lambda_{n}<\lambda_{n+1}<\lambda_{*}$ so that $\lambda_{n} \rightarrow \lambda_{*}$, $k_{n} \equiv k\left(\lambda_{n}\right)<0$, and

$$
k_{n} \rightarrow k\left(\lambda_{*}\right)=0 .
$$

Therefore, $k_{n}$ are negative eigenvalues. By Lemma 2.2. we get a sequence $\phi_{n} \in H^{2}$ such that

$$
A_{\lambda_{n}} \phi_{n}=k_{n} \phi_{n}
$$

with $k_{n}<0, k_{n} \rightarrow 0$ and $\lambda_{n} \rightarrow \lambda_{0}>0$, as $n \rightarrow \infty$. Recall $\chi$ the cutoff function of the support of $f_{0}(E)$ such that $\chi \equiv 1$ for $f_{0}(E)>0$. We claim that $\chi \phi_{n}$ is a nonzero function for any $n$. Suppose otherwise, $\chi \phi_{n} \equiv 0$, then from the equation (30) we have $\left(-\Delta-k_{n}\right) \phi_{n}=0$ which implies that $\phi_{n}=0$, a contradiction. Thus we can normalize $\phi_{n}$ by $\left\|\chi \phi_{n}\right\|_{2}=1$. Taking inner product of (30) with $\phi_{n}$ and integrating by parts, we have

$$
\begin{aligned}
\left\|\nabla \phi_{n}\right\|_{2}^{2} & \leq-4 \pi \iint f_{0}^{\prime}(E) \phi_{n}^{2} d v d x+\iint 4 \pi f_{0}^{\prime}(E) \int_{-\infty}^{0} \lambda_{n} e^{\lambda_{n} s} \phi_{n}(X(s ; x, v)) d s \phi_{n}(x) d x \\
& =-4 \pi \iint f_{0}^{\prime}(E)\left(\chi \phi_{n}\right)^{2} d v d x \\
& +\iint 4 \pi f_{0}^{\prime}(E) \int_{-\infty}^{0} \lambda_{n} e^{\lambda_{n} s}\left(\chi \phi_{n}\right)(X(s ; x, v)) d s\left(\chi \phi_{n}\right)(x) d x \\
& \leq 8 \pi\left|\int f_{0}^{\prime}(E) d v\right|_{\infty}\left\|\chi \phi_{n}\right\|_{2}^{2} .
\end{aligned}
$$

Here in the second equality above, we use the fact $\chi=1$ on the support of $f_{0}^{\prime}(E)\left(f_{0}(E)\right)$ and that $\left(\chi \phi_{n}\right)(X(s ; x, v))=\phi_{n}(X(s ; x, v) \chi$ due to the invariance of the support under the trajectory flow, as in (15). In the last inequality, we use the same estimate as in (16). Thus,

$$
\sup _{n}\left\|\phi_{n}\right\|_{L^{6}} \leq C \sup _{n}\left\|\nabla \phi_{n}\right\|_{2}<C^{\prime},
$$

for some constant $C^{\prime}$ independent of $n$. Then there exists $\phi \in L^{6}$ and $\nabla \phi \in L^{2}$ such that

$$
\phi_{n} \rightarrow \phi \text { weakly in } L^{6}, \quad \text { and } \nabla \phi_{n} \rightarrow \nabla \phi \text { weakly in } L^{2} \text {. }
$$


This implies that $\chi \phi_{n} \rightarrow \chi \phi$ strongly in $L^{2}$. Therefore $\|\chi \phi\|_{2}=1$ and thus $\phi \neq 0$. It is easy to show that $\phi$ is a weak solution of $A_{\lambda_{0}} \phi=0$ or

$$
-\Delta \phi=-\left[4 \pi \int f_{0}^{\prime}(E) d v\right] \phi+4 \pi f_{0}^{\prime}(E) \int_{-\infty}^{0} \lambda_{0} e^{\lambda_{0} s} \phi(X(s ; x, v)) d s d v=\rho .
$$

We have that

$$
\begin{aligned}
\int \rho d x & =-4 \pi \iint f_{0}^{\prime}(E) \phi(x) d x d v+\int_{-\infty}^{0} \lambda_{0} e^{\lambda_{0} s} \iint 4 \pi f_{0}^{\prime}(E) \phi(X(s ; x, v)) d x d v d s \\
& =-4 \pi \iint f_{0}^{\prime}(E) \phi(x) d x d v+\int_{-\infty}^{0} \lambda_{0} e^{\lambda_{0} s} \iint 4 \pi f_{0}^{\prime}(E) \phi(x) d x d v d s=0
\end{aligned}
$$

and by (31) $\rho$ has compact support in $S_{x}$, the $x$-support of $f_{0}(E)$. Therefore from the formula $\phi(x)=\int \frac{\rho(y)}{|x-y|} d y$, we have

$$
\phi(x)=\int \frac{\rho(y)}{|x-y|} d y=\int \frac{\rho(y)}{|x-y|} d y-\int \frac{\rho(y)}{|x|} d y=O\left(|x|^{-2}\right)
$$

for $x$ large, and thus $\phi \in L^{2}$. By elliptic regularity, $\phi \in H^{2}$. We define $f(x, v)$ by (14), then $f \in L^{\infty}$ with the compact support in $S$. Now we show that $e^{\lambda_{0} t}[f, \phi]$ is a weak solution to the linearized Vlasov-Poisson system. Since $\phi$ satisfies the Poisson equation (31), we only need to show that $f$ satisfies the linearized Vlasov equation (12) weakly. For that, we take any $g \in C_{c}^{1}\left(\mathbb{R}^{3} \times \mathbb{R}^{3}\right)$, and

$$
\begin{aligned}
& \iint_{\mathbb{R}^{3} \times \mathbb{R}^{3}}(D g) f d x d v \\
& =\iint_{\mathbb{R}^{3} \times \mathbb{R}^{3}}(D g)\left(f_{0}^{\prime}(E) \phi(x)\right) d x d v-\iint_{\mathbb{R}^{3} \times \mathbb{R}^{3}}(D g) f_{0}^{\prime}(E) \int_{-\infty}^{0} \lambda_{0} e^{\lambda_{0} s} \phi(X(s ; x, v)) d s d x d v \\
& =I+I I .
\end{aligned}
$$

Since $D$ is skew-adjoint, the first term is

$$
I=-\iint_{\mathbb{R}^{3} \times \mathbb{R}^{3}} g D\left(f_{0}^{\prime}(E) \phi\right) d x d v=-\iint_{\mathbb{R}^{3} \times \mathbb{R}^{3}} f_{0}^{\prime}(E) g D \phi d x d v .
$$


For the second term,

$$
\begin{aligned}
I I & =-\int_{-\infty}^{0} \lambda_{0} e^{\lambda_{0} s} \iint_{\mathbb{R}^{3} \times \mathbb{R}^{3}} f_{0}^{\prime}(E) D g(x, v) \phi(X(s ; x, v)) d x d v d s \\
& =-\int_{-\infty}^{0} \lambda_{0} e^{\lambda_{0} s} \iint_{\mathbb{R}^{3} \times \mathbb{R}^{3}} f_{0}^{\prime}(E)(D g)(X(-s), V(-s)) \phi(x) d x d v d s \\
& =-\iint_{\mathbb{R}^{3} \times \mathbb{R}^{3}} f_{0}^{\prime}(E) \int_{-\infty}^{0} \lambda_{0} e^{\lambda_{0} s}\left(-\frac{d}{d s} g(X(-s), V(-s))\right) d s \phi(x) d x d v \\
& =\iint_{\mathbb{R}^{3} \times \mathbb{R}^{3}} f_{0}^{\prime}(E)\left\{\lambda_{0} g(x, v)-\int_{-\infty}^{0} \lambda_{0}^{2} e^{\lambda_{0} s} g(X(-s), V(-s)) d s\right\} \phi(x) d x d v \\
& =\iint_{\mathbb{R}^{3} \times \mathbb{R}^{3}}\left\{f_{0}^{\prime}(E) \lambda_{0} \phi(x)-f_{0}^{\prime}(E) \int_{-\infty}^{0} \lambda_{0}^{2} e^{\lambda_{0} s} \phi(X(s), V(s)) d s\right\} g(x, v) d x d v \\
& =\lambda_{0} \iint_{\mathbb{R}^{3} \times \mathbb{R}^{3}}\left\{f_{0}^{\prime}(E) \phi(x)-f_{0}^{\prime}(E) \int_{-\infty}^{0} \lambda_{0} e^{\lambda_{0} s} \phi(X(s), V(s)) d s\right\} g d x d v \\
& =. \lambda_{0} \iint_{\mathbb{R}^{3} \times \mathbb{R}^{3}} f g d x d v .
\end{aligned}
$$

Thus we have

$$
\iint_{\mathbb{R}^{3} \times \mathbb{R}^{3}}(D g) f d x d v=\iint_{\mathbb{R}^{3} \times \mathbb{R}^{3}}\left(\lambda_{0} f-f_{0}^{\prime}(E) D \phi\right) g d x d v
$$

which implies that $f$ is a weak solution to the linearized Vlasov equation

$$
\lambda_{0} f+D f=f_{0}^{\prime}(E) v \cdot \nabla_{x} \phi .
$$

Remark 2. Consider an anisotropic spherical galaxy with $f_{0}(x, v)=f_{0}\left(E, L^{2}\right)$.

For a radial symmetric growing mode $e^{\lambda t}(\phi, f)$ with $\phi=\phi(|x|)$ and $f=f\left(|x|, E, L^{2}\right)$. The linearized Vlasov equation (11) becomes

$$
\begin{aligned}
& \lambda f+v \cdot \nabla_{x} f-\nabla_{x} U_{0} \cdot \nabla_{v} f \\
= & \nabla_{x} \phi \cdot \nabla_{v} f_{0}=\nabla_{x} \phi \cdot\left(\frac{\partial f_{0}}{\partial E} v+\frac{\partial f_{0}}{\partial L^{2}} \nabla_{v}\left(|x \times v|^{2}\right)\right) \\
= & \phi^{\prime}(|x|) \frac{x}{|x|} \cdot\left(\frac{\partial f_{0}}{\partial E} v+2 \frac{\partial f_{0}}{\partial L^{2}}[(x \times v) \times x]\right)=\frac{\partial f_{0}}{\partial E} v \cdot \nabla_{x} \phi,
\end{aligned}
$$

which is of the same form as in the isotropic case (20). So by the same proof of Theorem 1.1, we also get an instability criterion for radial perturbations of anisotropic galaxy, in terms of the quadratic form (18) with $f_{0}^{\prime}(E)$ being replaced by $\frac{\partial f_{0}}{\partial E}$.

\section{Nonlinear Stability of the King's Model}

In the second half of the article, we investigate the nonlinear stability of the King model (8). We first establish:

Lemma 3.1. Consider spherical models $f_{0}=f_{0}(E)$ with $f_{0}^{\prime}<0$. The operator $A_{0}: H_{r}^{2} \rightarrow L_{r}^{2}$

$$
A_{0} \phi=-\Delta \phi+\left[4 \pi \int f_{0}^{\prime} d v\right] \phi-4 \pi \int f_{0}^{\prime} \mathcal{P} \phi d v
$$


is positive, where $H_{r}^{2}$ and $L_{r}^{2}$ are spherically symmetric subspaces of $H^{2}$ and $L^{2}$, and the projection $\mathcal{P} \phi$ is defined by (27). Moreover, for $\phi \in H_{r}^{2}$ we have

$$
\left(A_{0} \phi, \phi\right) \geq \varepsilon\left(|\nabla \phi|_{2}^{2}+|\phi|_{2}^{2}\right)
$$

for some constant $\varepsilon>0$.

Proof. Define $k_{0}=\inf \left(A_{0} \phi, \phi\right) /(\phi, \phi)$. We want to show that $k_{0}>0$. First, by using the compact embedding of $H_{r}^{2} \hookrightarrow L_{r}^{2}$ it is easy to show that the minimum can be obtained and $k_{0}$ is the lowest eigenvalue. Let $A_{0} \phi_{0}=k_{0} \phi_{0}$ with $\phi_{0} \in H_{r}^{2}$ and $\left\|\phi_{0}\right\|_{2}=1$. The fact that $k_{0} \geq 0$ follows immediately from Theorem 1.1 and the nonexistence of radial modes $([9,22])$ for monotone spherical models. The proof of $k_{0}>0$ is more delicate. For that, we relate the quadratic form $\left(A_{0} \phi, \phi\right)$ to the Antonov functional (4). We define $D=v \cdot \partial_{x}-\nabla_{x} U_{0} \cdot \nabla_{v}$ to be the generator of the unitary group $U(s): L_{\left|f_{0}^{\prime}\right|}^{2, r} \rightarrow L_{\left|f_{0}^{\prime}\right|}^{2, r}$ defined by $U(s) m=m(X(s ; x, v), V(s ; x, v))$. Here $L_{\left|f_{0}^{\prime}\right|}^{2, r}$ is the spherically symmetric subspace of $L_{\left|f_{0}^{\prime}\right|}^{2}$, which is preserved under the flow mapping $U(s)$. By the definition of $\mathcal{P} \phi$, we have $\phi_{0}-\mathcal{P} \phi_{0} \perp \operatorname{ker} D$. By Stone theorem $i D$ is self-adjoint and in particular $D$ is closed. Therefore by the closed range theorem (40]), we have $(\operatorname{ker} D)^{\perp}=R(D)$, where $R(D)$ is the range of $D$. So there exists $h \in L_{\left|f_{0}^{\prime}\right|}^{2, r}$ such that $D h=\phi_{0}-\mathcal{P} \phi_{0}$. Moreover, since $\phi_{0}-\mathcal{P} \phi_{0}$ is even in $v$ and the operator $D$ reverses the parity in $v$, the function $h$ is odd in $v$. Define $f^{-}=f_{0}^{\prime} h$. We have

$$
\begin{aligned}
k_{0}= & \left(A_{0} \phi_{0}, \phi_{0}\right)=\int\left|\nabla \phi_{0}\right|^{2} d x+4 \pi \iint f_{0}^{\prime}\left(\phi_{0}-\mathcal{P} \phi_{0}\right)^{2} d x d v \\
= & \int\left|\nabla \phi_{0}\right|^{2} d x-8 \pi \iint\left|f_{0}^{\prime}\right|\left(\phi_{0}-\mathcal{P} \phi_{0}\right) \phi_{0} d x d v \\
& +4 \pi \iint\left|f_{0}^{\prime}\right|\left(\phi_{0}-\mathcal{P} \phi_{0}\right)^{2} d x d v \\
= & 4 \pi\left(\iint \frac{\left|D f^{-}\right|^{2}}{\left|f_{0}^{\prime}\right|} d x d v+2 \int \phi_{0} \int D f^{-} d v d x+\frac{1}{4 \pi} \int\left|\nabla \phi_{0}\right|^{2} d x\right) \\
= & 4 \pi\left(\iint \frac{\left|D f^{-}\right|^{2}}{\left|f_{0}^{\prime}\right|} d x d v+\frac{1}{2 \pi} \int \phi_{0} \Delta \phi^{-} d x+\frac{1}{4 \pi} \int\left|\nabla \phi_{0}\right|^{2} d x\right) \\
= & 4 \pi\left(\iint \frac{\left|D f^{-}\right|^{2}}{\left|f_{0}^{\prime}\right|} d x d v+\frac{1}{4 \pi} \int\left(\left|\nabla \phi_{0}\right|^{2}-2 \nabla \phi_{0} \cdot \nabla \phi^{-}\right) d x\right) \\
\geq & 4 \pi\left(\iint \frac{\left|D f^{-}\right|^{2}}{\left|f_{0}^{\prime}\right|} d x d v-\frac{1}{4 \pi} \int\left|\nabla \phi^{-}\right|^{2} d x\right)
\end{aligned}
$$

where $\Delta \phi^{-}=4 \pi \int D f^{-} d v$. Notice that the last expression above is the Antonov functional $4 \pi H\left(f^{-}, f^{-}\right)$. Since $f^{-}$is spherical symmetric and odd in $v$, we have $H\left(f^{-}, f^{-}\right)>0$ by the proof in [22] which was further clarified in [33] and [21]. Therefore we get $k_{0}>0$ as desired and $\left(A_{0} \phi, \phi\right) \geq k_{0}|\phi|_{2}^{2}$. 
To get the estimate (32), we rewrite

$$
\begin{aligned}
\left(A_{0} \phi, \phi\right) & =\varepsilon\left(\int|\nabla \phi|^{2} d x+4 \pi \iint f_{0}^{\prime}(\phi-\mathcal{P} \phi)^{2} d x d v\right)+(1-\varepsilon)\left(A_{0} \phi, \phi\right) \\
& \geq \varepsilon \int|\nabla \phi|^{2} d x-4 \pi \varepsilon\|\phi-\mathcal{P} \phi\|_{L_{\left|f_{0}^{\prime}\right|}^{2}}^{2}+(1-\varepsilon) k_{0}|\phi|_{2}^{2} \\
& \geq \varepsilon \int|\nabla \phi|^{2} d x-8 \pi \varepsilon\|\phi\|_{L_{\left|f_{0}^{\prime}\right|}^{2}}^{2}+(1-\varepsilon) k_{0}|\phi|_{2}^{2}\left(\text { since }\|\mathcal{P}\|_{\left.L_{\left|f_{0}^{\prime}\right|}^{2} \rightarrow L_{\left|f_{0}^{\prime}\right|}^{2} \leq 1\right)}\right. \\
& \geq \varepsilon \int|\nabla \phi|^{2} d x+\left((1-\varepsilon) k_{0}-C \varepsilon\right)|\phi|_{2}^{2} \geq \varepsilon\left(\int|\nabla \phi|^{2} d x+|\phi|_{2}^{2}\right)
\end{aligned}
$$

if $\varepsilon$ is small enough.

Next, we will approximate the $\operatorname{ker} D$ by a finite dimensional approximation. Let $\left\{\xi_{i}(E, L)=\alpha_{i}(E) \beta_{i}(L)\right\}_{i=1}^{\infty}$ be a smooth orthogonal basis for the subspace ker $D=$ $\{g(E, L)\} \subset L_{\left|f_{0}^{\prime}\right|}^{2, r}$. Define the finite-dimensional projection operator $\mathcal{P}_{N}: L_{\left|f_{0}^{\prime}\right|}^{2, r} \rightarrow$ $L_{\left|f_{0}^{\prime}\right|}^{2, r}$ by

$$
\mathcal{P}_{N} h \equiv \sum_{i=1}^{N}\left(h, \xi_{i}\right)_{\left|f_{0}^{\prime}\right|} \xi_{i}
$$

and the operator $A^{N}: H_{r}^{2} \rightarrow L_{r}^{2}$ by

$$
A^{N} \phi=-\Delta \phi+\left[4 \pi \int f_{0}^{\prime} d v\right] \phi-4 \pi \int f_{0}^{\prime} \mathcal{P}_{N} \phi d v .
$$

Lemma 3.2. There exists $K, \delta_{0}>0$ such that when $N>K$ we have

$$
\left(A^{N} \phi, \phi\right) \geq \delta_{0}|\nabla \phi|_{2}^{2}
$$

for any $\phi \in H_{r}^{2}$.

Proof. First we have $A^{N} \rightarrow A_{0}$ strongly in $L^{2}$. In deed, for any $\phi \in H_{r}^{2}$,

$$
\left\|A^{N} \phi-A_{0} \phi\right\|_{2}=\left\|\int 4 \pi f_{0}^{\prime}\left(\mathcal{P}_{N} \phi-\mathcal{P} \phi\right) d v\right\|_{2} \leq C\left\|\mathcal{P}_{N} \phi-\mathcal{P} \phi\right\|_{L_{\left|f_{0}^{\prime}\right|}^{2}} \rightarrow 0
$$

as $N \rightarrow \infty$.We claim that for $N$ sufficiently large, the lowest eigenvalue of $A^{N}$ is at least $k_{0} / 2$ where $k_{0}>0$ is the lowest eigenvalue of $A_{0}$. Suppose otherwise, then there exists a sequence $\left\{\lambda_{n}\right\}$ and $\left\{\phi_{n}\right\} \subset H_{r}^{2}$ with $\lambda_{n}<k_{0} / 2,\left\|\phi_{n}\right\|_{2}=$ 1 and $A^{n} \phi_{n}=\lambda_{n} \phi_{n}$. This implies that $\Delta \phi_{n}$ is uniformly bounded in $L^{2}$, by elliptic estimate we have $\left\|\phi_{n}\right\|_{H^{2}} \leq C$ for some constant $C$ independent of $n$. Therefore there exists $\phi_{0} \in H_{r}^{2}$ such that $\phi_{n} \rightarrow \phi_{0}$ weakly in $H_{r}^{2}$. By the compact embedding of $H_{r}^{2} \hookrightarrow L_{r}^{2}$, we have $\phi_{n} \rightarrow \phi_{0}$ strongly in $L_{r}^{2}$ and $\left\|\phi_{0}\right\|_{2}=1$. The strong convergence of $A^{n} \phi_{0} \rightarrow A_{0} \phi_{0}$ implies that

$$
A^{n} \phi_{n} \rightarrow A_{0} \phi_{0}
$$

weakly in $L^{2}$. Let $\lambda_{n} \rightarrow \lambda_{0} \leq k_{0} / 2$, then we have $A_{0} \phi_{0}=\lambda_{0} \phi_{0}$, a contradiction. Therefore we have $\left(A^{N} \phi, \phi\right) \geq k_{0} / 2|\phi|_{2}^{2}$ for $\phi \in H_{r}^{2}$, when $N$ is large enough. The estimate (34) is by the same proof of (32) in Lemma 3.1 . 
Recalling (8) with $f_{0}=\left[e^{E_{0}-E}-1\right]_{+}$and $Q_{0}(f)=(f+1) \ln (f+1)-f$, we further define functionals (related to the finite dimensional approximation of $\operatorname{ker} D$ ) as

$$
\begin{aligned}
A_{i}(f) & \equiv \int_{0}^{f} \alpha_{i}\left(-\ln (s+1)+E_{0}\right) d s, \\
Q_{i}(f, L) & \equiv A_{i}(f) \beta_{i}(L), \text { for } 1 \leq i \leq N .
\end{aligned}
$$

for $1 \leq i \leq N$. Clearly,

$$
\partial_{1} Q_{i}\left(f_{0}, L\right)=\alpha_{i}\left(-\ln \left(f_{0}+1\right)+E_{0}\right) \beta_{i}(L)=\alpha_{i}(E) \beta_{i}(L)=\xi_{i}(E, L),
$$

where $\left\{\xi_{i}(E, L)\right\}_{i=1}^{N}$ are used to define $\mathcal{P}_{N}$ in Lemma 3.2. Define the Casimir functional $\left(E_{0}<0\right)$

$$
I(f)=\int\left[Q_{0}(f)+\frac{1}{2}|v|^{2} f-E_{0} f\right] d x d v-\frac{1}{8 \pi} \int|\nabla \phi|^{2} d x
$$

which is invariant of the nonlinear Vlasov-Poisson system. We introduce additional $N$ invariants

$$
J_{i}(f, L) \equiv \int Q_{i}(f, L) d x d v
$$

for $1 \leq i \leq N$. We define $\Omega$ to be the support of $f_{0}(E)$. We first consider

$$
\begin{aligned}
I(f)-I\left(f_{0}\right)= & \int\left[Q_{0}(f)-Q_{0}\left(f_{0}\right)+\frac{1}{2}|v|^{2}\left(f-f_{0}\right)-E_{0}\left(f-f_{0}\right)\right] d x d v \\
& -\frac{1}{4 \pi} \int \nabla U_{0} \cdot \nabla\left(U-U_{0}\right)-\frac{1}{8 \pi} \int\left|\nabla\left(U-U_{0}\right)\right|^{2} d x \\
= & \int\left[Q_{0}(f)-Q_{0}\left(f_{0}\right)+\left(E-E_{0}\right)\left(f-f_{0}\right)\right] d x d v-\frac{1}{8 \pi} \int\left|\nabla\left(U-U_{0}\right)\right|^{2} d x .
\end{aligned}
$$

We define

$$
g=f-f_{0}, \quad \phi=U-U_{0}
$$

and

$$
g_{\text {in }} \equiv\left(f-f_{0}\right) \mathbf{1}_{\Omega}, \quad g_{\text {out }} \equiv\left(f-f_{0}\right) \mathbf{1}_{\Omega^{c}}, \quad \Delta \phi_{\text {in }} \equiv \int g_{\text {in }}, \quad \Delta \phi_{\text {out }} \equiv \int g_{\text {out }} .
$$

And we define the distance function for nonlinear stability as

$$
\begin{aligned}
d\left(f, f_{0}\right) & \equiv\left\{\iint\left[Q_{0}\left(g_{\text {in }}+f_{0}\right)-Q_{0}\left(f_{0}\right)+\left(E-E_{0}\right) g_{\text {in }}\right] d x d v\right\}+\frac{1}{8 \pi} \int\left|\nabla \phi_{\text {in }}\right|^{2} d x \\
& +\left\{\iint Q_{0}\left(g_{\text {out }}\right) d x d v+\int_{E \geq E_{0}}\left(E-E_{0}\right) g_{\text {out }} d x d v\right\} \\
& =d_{\text {in }}+\frac{1}{8 \pi} \int\left|\nabla \phi_{\text {in }}\right|^{2} d x+d_{\text {out }},
\end{aligned}
$$

for which each term is non-negative. We therefore split:

$$
\begin{aligned}
& I(f)-I\left(f_{0}\right) \\
& =\left\{\int\left[Q_{0}\left(f_{0}+g_{\text {in }}\right)-Q_{0}\left(f_{0}\right)+\left(E-E_{0}\right) g_{\text {in }}\right] d x d v-\frac{1}{8 \pi} \int\left|\nabla \phi_{\text {in }}\right|^{2} d x\right\}+ \\
& \left\{\int Q_{0}\left(g_{\text {out }}\right) d x d v+\int_{E \geq E_{0}}\left(E-E_{0}\right) g_{\text {out }} d x d v-\frac{1}{8 \pi} \int\left|\nabla \phi_{\text {out }}\right|^{2} d x-\frac{1}{4 \pi} \int \nabla \phi_{\text {out }} \cdot \nabla \phi_{\text {in }} d x\right\} \\
& =I_{\text {in }}+I_{\text {out }} .
\end{aligned}
$$


In the estimates below, we use $C, C^{\prime}, C^{\prime \prime}$ to denote general constants depending only on $f_{0}$ and quantities like $\|f(t)\|_{L^{p}}(p \in[1,+\infty])$ which equals $\|f(0)\|_{L^{p}}$ and therefore always under control. We first estimate $\left\|\nabla \phi_{\text {out }}\right\|_{2}^{2}$ to be of higher order of $d$, which also implies that $\int \nabla \phi_{\text {out }} \cdot \nabla \phi_{\text {in }} d x$ is of higher order of $d$.

Lemma 3.3. For $\varepsilon>0$ sufficiently small, we have

$$
\int\left|\nabla \phi_{\text {out }}\right|^{2} d x \leq C\left(\varepsilon d\left(f, f_{0}\right)+\frac{1}{\varepsilon^{5 / 3}}\left[d\left(f, f_{0}\right)\right]^{5 / 3}\right) .
$$

Proof. In fact, since

$$
\begin{aligned}
\int\left|\nabla \phi_{\text {out }}\right|^{2} d x & \leq C\left\|\int g_{\text {out }} d v\right\|_{L^{6 / 5}}^{2} \\
& \leq C\left\|\int g_{\text {out }} \mathbf{1}_{E_{0} \leq E \leq E_{0}+\varepsilon} d v\right\|_{L^{6 / 5}}^{2}+C\left\|\int g_{\text {out }} \mathbf{1}_{E>E_{0}+\varepsilon} d v\right\|_{L^{6 / 5}}^{2} .
\end{aligned}
$$

The first term is bounded by

$$
\begin{aligned}
& {\left[\int\left[\int g_{\text {out }}^{2} d v\right]^{3 / 5}\left[\int \mathbf{1}_{E_{0} \leq E \leq E_{0}+\varepsilon} d v\right]^{3 / 5} d x\right]^{5 / 3}} \\
& \leq\left[\int g_{\text {out }}^{2} d v d x\right] \times\left[\int\left[\int \mathbf{1}_{E_{0} \leq E \leq E_{0}+\varepsilon} d v\right]^{3 / 2} d x\right]^{2 / 3} \\
& \leq C \varepsilon\left[\int g_{\text {out }}^{2} d v d x\right] \leq C \varepsilon\left[\int g_{\text {out }}^{2} d v d x\right] \\
& \leq C \varepsilon d\left(f, f_{0}\right) .
\end{aligned}
$$

In the above estimates, we use that $\iint Q_{0}\left(g_{\text {out }}\right) d v d x \geq c \int g_{\text {out }}^{2} d v d x$ and

$$
\int \mathbf{1}_{E_{0} \leq E \leq E_{0}+\varepsilon} d v \leq C \varepsilon
$$

which can be checked by an explicit computation when $\varepsilon>0$ is sufficiently small such that $E_{0}+\varepsilon \leq 0$.

On the other hand, by the standard estimates (see [12, P. 120-121])

$$
\begin{aligned}
& \left\|\int g_{\text {out }} \mathbf{1}_{E>E_{0}+\varepsilon} d v\right\|_{L^{6 / 5}}^{2} \\
& \leq\left[\iint g_{\text {out }} \mathbf{1}_{E>E_{0}+\varepsilon} d x d v\right]^{\frac{7}{6}} \times\left[\iint|v|^{2} g_{\text {out }} \mathbf{1}_{E>E_{0}+\varepsilon} d x d v\right]^{\frac{1}{2}} \\
& \leq\left[\frac{1}{\varepsilon} \iint\left(E-E_{0}\right) g_{\text {out }} \mathbf{1}_{E>E_{0}+\varepsilon} d x d v\right]^{\frac{7}{6}} \\
& \times\left[\iint\left(E-E_{0}\right) g_{\text {out }} \mathbf{1}_{E>E_{0}+\varepsilon} d x d v+2 \sup \left|U_{0}\right| \iint g_{\text {out }} \mathbf{1}_{E>E_{0}+\varepsilon} d x d v\right]^{\frac{1}{2}} \\
& \leq\left(\frac{1}{\varepsilon} d\right)^{\frac{7}{6}}\left(d+\frac{2 \sup \left|U_{0}\right|}{\varepsilon} d\right)^{\frac{1}{2}} \leq \frac{C}{\varepsilon^{5 / 3}} d^{5 / 3} .
\end{aligned}
$$


By Lemma 3.3 we have

$$
\begin{aligned}
\left|\int \nabla \phi_{\text {out }} \cdot \nabla \phi_{\text {in }} d x\right| & \leq\left\|\nabla \phi_{\text {out }}\right\|_{2}\left\|\nabla \phi_{\text {in }}\right\|_{2} \\
& \leq C\left(\varepsilon^{1 / 3} d\left(f, f_{0}\right)+\frac{1}{\varepsilon^{5 / 6}}\left[d\left(f, f_{0}\right)\right]^{4 / 3}\right)
\end{aligned}
$$

and therefore for $\varepsilon$ sufficiently small,

$$
I_{\text {out }} \geq d_{\text {out }}-C\left(\varepsilon^{1 / 3} d\left(f, f_{0}\right)+\frac{1}{\varepsilon^{5 / 6}}\left[d\left(f, f_{0}\right)\right]^{4 / 3}+\frac{1}{\varepsilon^{5 / 3}}\left[d\left(f, f_{0}\right)\right]^{5 / 3}\right) .
$$

To estimate $I_{\text {in }}$, we split it into three parts:

$$
\begin{aligned}
& \tau\left\{\int\left[Q_{0}\left(f_{0}+g_{\text {in }}\right)-Q_{0}\left(f_{0}\right)+\left(E-E_{0}\right) g_{\text {in }}+\phi_{\text {in }} g_{\text {in }}\right] d x d v+\frac{1}{8 \pi} \int\left|\nabla \phi_{\text {in }}\right|^{2} d x\right\}+ \\
& (1-\tau)\left\{\int\left[Q_{0}\left(f_{0}+g_{\text {in }}\right)-Q_{0}\left(f_{0}\right)+\left(E-E_{0}\right) g_{\text {in }}+\left(I-P_{N}\right) \phi_{\text {in }} g_{\text {in }}\right] d x d v+\frac{1}{8 \pi} \int\left|\nabla \phi_{\text {in }}\right|^{2} d x\right\} \\
& +(1-\tau) \int P_{N} \phi_{\text {in }} g_{\text {in }} d x d v \\
& (37) \\
& =I_{\text {in }}^{1}+I_{\text {in }}^{2}+I_{\text {in }}^{3},
\end{aligned}
$$

where $\Delta \phi_{\text {in }}=4 \pi \int g_{\text {in }} d v$. We estimate each term in the following lemmas.

\section{Lemma 3.4.}

$$
I_{i n}^{1} \geq \frac{\tau}{2} d_{i n}-C \tau \int\left|\nabla \phi_{i n}\right|^{2} d x
$$

Proof. In fact, since the integration region $\Omega$ is finite, we have

$$
\begin{aligned}
I_{\text {in }}^{1}=\tau & {\left[\iint\left[Q_{0}\left(f_{0}+g_{\text {in }}\right)-Q_{0}\left(f_{0}\right)+\left(E-E_{0}\right) g_{\text {in }}+\phi_{\text {in }} g_{\text {in }}\right] d x d v+\frac{1}{8 \pi} \int\left|\nabla \phi_{\text {in }}\right|^{2} d x\right] } \\
& \geq \tau \iint\left[Q_{0}\left(f_{0}+g_{\text {in }}\right)-Q_{0}\left(f_{0}\right)+\left(E-E_{0}\right) g_{\text {in }}\right] d x d v-C \tau\left\|\phi_{\text {in }}\right\| L_{L^{6}}\left\|g_{\text {in }}\right\|_{L^{6 / 5}} \\
& \geq \tau \iint\left[Q_{0}\left(f_{0}+g_{\text {in }}\right)-Q_{0}\left(f_{0}\right)+\left(E-E_{0}\right) g_{\text {in }}\right] d x d v-C^{\prime} \tau\left\|\nabla \phi_{\text {in }}\right\|\left\|_{L^{2}}\right\| g_{\text {in }} \|_{2} \\
& \geq \frac{\tau}{2} d_{\text {in }}-C^{\prime \prime} \tau\left\|\nabla \phi_{\text {in }}\right\|_{2}^{2},
\end{aligned}
$$

since

$$
d_{\text {in }}=\int\left[Q_{0}\left(f_{0}+g_{\text {in }}\right)-Q_{0}\left(f_{0}\right)+\left(E-E_{0}\right) g_{\text {in }}\right] d x d v \geq C\left\|g_{\text {in }}\right\|_{2}^{2} .
$$

To estimate $I_{\text {in }}^{2}$, we need the following pointwise duality lemma from elementary calculus.

Lemma 3.5. For any $c$, and any $h$, we have

$$
g_{c, f_{0}}(h)=Q_{0}\left(h+f_{0}\right)-Q_{0}\left(f_{0}\right)-Q_{0}^{\prime}\left(f_{0}\right) h-c h \geq\left(f_{0}+1\right)\left(1+c-e^{c}\right) .
$$

Proof. Direct computation yields that the minimizer $f_{c}$ of $g_{c, f_{0}}(h)$ satisfies the Euler-Lagrange equation

$$
\ln \left(f_{c}+f_{0}+1\right)-\ln \left(f_{0}+1\right)-c=0,
$$


SO

$$
f_{c}=\left(f_{0}+1\right)\left(e^{c}-1\right) .
$$

Thus by using the Euler-Lagrange equation, we deduce

$$
\begin{aligned}
\min g_{c, f_{0}}(h) & =g_{c, d}\left(f_{c}\right) \\
& =\left(f_{c}+f_{0}+1\right) \ln \left(1+f_{c}+f_{0}\right) \\
& -\left(f_{0}+1\right) \ln \left(1+f_{0}\right)-\left[1+\ln \left(f_{0}+1\right)\right] f_{c}-c f_{c} \\
& =\left(f_{c}+f_{0}+1\right)\left[\ln \left(1+f_{c}+f_{0}\right)-\ln \left(f_{0}+1\right)-c\right] \\
& +f_{c} \ln \left(1+f_{0}\right)+c\left(f_{0}+1\right)-\left[1+\ln \left(f_{0}+1\right)\right] f_{c} \\
& =\left(f_{0}+1\right)\left(1+c-e^{c}\right) .
\end{aligned}
$$

\section{Lemma 3.6.}

$$
I_{\text {in }}^{2} \geq \frac{(1-\tau) \delta_{0}}{8 \pi} \int\left|\nabla \phi_{i n}\right|^{2} d x-C e^{C^{\prime} d^{\frac{1}{2}}} d^{\frac{3}{2}} .
$$

Proof. Recall (37). By using Lemma 3.5 for $c=-\left(\phi_{\text {in }}-P_{N} \phi_{\text {in }}\right)$ and using the Taylor expansion, we have

$$
\begin{aligned}
& I_{\mathrm{in}}^{2}=(1-\tau) \iint\left[Q_{0}\left(f_{0}+g_{\mathrm{in}}\right)-Q_{0}\left(f_{0}\right)+\left(E-E_{0}\right) g_{\mathrm{in}}+\left(\phi_{\mathrm{in}}-P_{N} \phi_{\mathrm{in}}\right) f_{\mathrm{in}}\right] d x d v \\
& +\frac{1}{8 \pi}(1-\tau) \int\left|\nabla \phi_{\text {in }}\right|^{2} d x \\
& \geq \frac{1}{8 \pi}(1-\tau) \int\left|\nabla \phi_{\mathrm{in}}\right|^{2} d x+(1-\tau) \iint\left(f_{0}+1\right) \mathbf{1}_{\Omega}\left(1+\phi_{\mathrm{in}}-P_{N} \phi_{\mathrm{in}}-e^{\phi_{\mathrm{in}}-P_{N} \phi_{\mathrm{in}}}\right) d x d v \\
& \geq \frac{1-\tau}{8 \pi}\left\{\int\left|\nabla \phi_{\text {in }}\right|^{2} d x-4 \pi \iint\left|f_{0}^{\prime}(E)\right|\left(\phi_{\text {in }}-P_{N} \phi_{\text {in }}\right)^{2} d x d v\right\} \\
& -C e^{\left|\phi_{\mathrm{in}}-P_{N} \phi_{\mathrm{in}}\right|_{\infty}} \iint\left|f_{0}^{\prime}(E)\right|\left|\phi_{\mathrm{in}}-P_{N} \phi_{\mathrm{in}}\right|^{3} d x d v \quad\left(\text { Note }\left(f_{0}(E)+1\right) \mathbf{1}_{\Omega}=\left|f_{0}^{\prime}(E)\right|\right)
\end{aligned}
$$

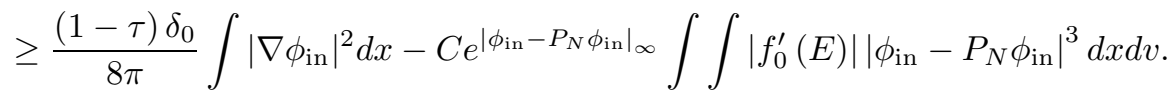

In the last line, we have used Lemma 3.2. To estimate the last term above and conclude our lemma, it suffices to show

$$
\left|\phi_{\text {in }}-P_{N} \phi_{\text {in }}\right|_{\infty} \leq C_{N} d^{\frac{1}{2}} .
$$

This follows from the facts that for the fixed $N$ smooth functions $\xi_{i}$, we have

$$
\left|P_{N} \phi_{\mathrm{in}}\right|_{\infty}=\left|\sum_{i=1}^{N}\left(\phi_{\mathrm{in}}, \xi_{i}\right)_{\left|f_{0}^{\prime}\right|} \xi_{i}\right|_{\infty} \leq C_{N}\left|\phi_{\mathrm{in}}\right|_{\infty},
$$

and since $\phi$ is spherically symmetric,

$$
\begin{aligned}
\left|\phi_{\text {in }}\right|(r) & =\left|\frac{1}{r} \int_{0}^{r} u^{2} \rho_{\text {in }}(u) d u+\int_{r}^{R} u \rho_{\text {in }}(u) d u\right| \\
& \leq C^{\prime} \sqrt{R}\left|\rho_{\text {in }}\right|_{2} \leq C^{\prime \prime}\left\|g_{\text {in }}\right\|_{2} \leq C_{N} d^{\frac{1}{2}}
\end{aligned}
$$

where $\rho_{\text {in }}=\int g_{\text {in }} d v$ and $R$ is the support radius of $\rho_{\text {in }}$. 
We now estimate the term $\iint P_{N} \phi_{\text {in }} f_{\text {in }} d x d v$, for which we use the additional invariants.

Lemma 3.7. For any $\varepsilon>0$, we have

$$
\left|I_{i n}^{3}\right| \leq C\left(d^{1 / 2}(0)+\varepsilon^{1 / 2} d^{1 / 2}+\frac{1}{\varepsilon} d\right) d^{1 / 2} .
$$

Proof. By the definition of $I_{\mathrm{in}}^{3}$ in (37), it suffices to estimate $\left(g_{\mathrm{in}}, \xi_{i}\right)$. We expand

$$
\begin{aligned}
& J_{i}(f, L)-J_{i}\left(f_{0}, L\right) \\
& =J_{i}\left(f_{0}+g_{\text {in }}, L\right)-J_{i}\left(f_{0}, L\right)+J_{i}\left(g_{\text {out }}, L\right) \\
& =\left(g_{\text {in }}, \xi_{i}\right)+O(d)+J_{i}\left(g_{\text {out }}, L\right) .
\end{aligned}
$$

Notice that

$$
\begin{aligned}
\left|J_{i}\left(g_{\text {out }}, L\right)\right| & \leq C\left\|g_{\text {out }}\right\|_{L^{1}} \leq C\left\|\mathbf{1}_{\left\{E_{0} \leq E \leq E_{0}+\varepsilon\right\}} g_{\text {out }}\right\|_{L^{1}}+C\left\|\mathbf{1}_{\left\{E \geq E_{0}+\varepsilon\right\}} g_{\text {out }}\right\|_{L^{1}} \\
& \leq \varepsilon^{1 / 2}\left\|g_{\text {out }}\right\|_{L^{2}}+\frac{C}{\varepsilon}\left\|\mathbf{1}_{\left\{E \geq E_{0}+\varepsilon\right\}}\left(E-E_{0}\right) g_{\text {out }}\right\|_{L^{1}} \leq C\left[\varepsilon^{1 / 2} d^{1 / 2}+\frac{1}{\varepsilon} d\right] .
\end{aligned}
$$

It thus follows that

$$
\begin{aligned}
\left|\left(g_{\text {in }}, \xi_{i}\right)\right| & \leq\left|J_{i}(f(0), L)-J_{i}\left(f_{0}, L\right)\right|+C\left[\varepsilon^{1 / 2} d^{1 / 2}+\frac{1}{\varepsilon} d\right] \\
& \leq C\left[d^{1 / 2}(0)+\varepsilon^{1 / 2} d^{1 / 2}+\frac{1}{\varepsilon} d\right] .
\end{aligned}
$$

Therefore

$$
\begin{aligned}
\left|I_{\text {in }}^{3}\right| & =(1-\tau)\left|\iint P_{N} \phi_{\text {in }} g_{\text {in }} d x d v\right|=\left|\iint\left(\sum_{i=1}^{N}\left(\phi_{\text {in }}, \xi_{i}\right)_{\mid f_{0}^{\prime}} \xi_{i}\right) g_{\text {in }} d x d v\right| \\
& \leq \sum_{i=1}^{N}\left|\left(\phi_{\text {in }}, \xi_{i}\right)_{\left|f_{0}^{\prime}\right|}\right|\left|\left(\xi_{i}, g_{\text {in }}\right)\right| \leq C^{\prime} \sum_{i=1}^{N}\left|\phi_{\text {in }}\right|_{\infty}\left|\left(\xi_{i}, g_{\text {in }}\right)\right| \\
& \leq C d^{1 / 2}\left[d^{1 / 2}(0)+\varepsilon^{1 / 2} d^{1 / 2}+\frac{1}{\varepsilon} d\right] .
\end{aligned}
$$

Now we prove the nonlinear stability of King model.

Proof of Theorem 1.2. The global existence of classical solutions of 3D VlasovPoisson system was shown in [34] for compactly supported initial data $f(0) \in C_{c}^{1}$. Let the unique global solution be $(f(t), \phi(t))$. Let $d(t)=d\left(f(t), f_{0}\right)$. Combining estimates (36), (38), (39) and (40), we have

$$
\begin{aligned}
I(f(0))-I\left(f_{0}\right) & =I(f(t))-I\left(f_{0}\right) \\
& \geq d_{\text {out }}+\frac{\tau}{2} d_{\text {in }}+\left(\frac{(1-\tau) \delta_{0}}{8 \pi}-C \tau\right) \int\left|\nabla \phi_{\text {in }}\right|^{2} d x \\
& -C\left(\varepsilon^{1 / 3} d(t)+\frac{1}{\varepsilon^{5 / 6}} d(t)^{4 / 3}+\frac{1}{\varepsilon^{5 / 3}} d(t)^{5 / 3}\right)-C e^{C^{\prime} d(t)^{\frac{1}{2}}} d(t)^{\frac{3}{2}} \\
& -C d(t)^{1 / 2}\left[d^{1 / 2}(0)+\varepsilon^{1 / 2} d(t)^{1 / 2}+\frac{1}{\varepsilon} d(t)\right] .
\end{aligned}
$$


Thus by choosing $\varepsilon$ and $\tau$ sufficiently small, there exists $\delta^{\prime}>0$ such that

$$
\begin{aligned}
I(f(0))-I\left(f_{0}\right) & \geq \delta^{\prime} d(t)-C\left(d(t)^{4 / 3}+d(t)^{5 / 3}+d(t)^{3 / 2}\right)-C e^{C^{\prime} d(t)^{\frac{1}{2}}} d(t)^{\frac{3}{2}} \\
& -C d(t)^{1 / 2} d^{1 / 2}(0) .
\end{aligned}
$$

It is easy to show that $I(f(0))-I\left(f_{0}\right) \leq C^{\prime \prime} d(0)$. Define the functions $y_{1}(x)=$ $\delta^{\prime} x^{2}-C e^{C^{\prime} x} x^{3}-C\left(x^{8 / 3}+x^{10 / 3}+x^{3}\right)$ and $y_{2}(x)=C d(0)^{1 / 2} x+C^{\prime \prime} d(0)$. Then above estimates implies that $y_{1}\left(d(t)^{1 / 2}\right) \leq y_{2}\left(d(t)^{1 / 2}\right)$. The function $y_{1}$ is increasing in $\left(0, x_{0}\right)$ where $x_{0}$ is the first maximum point. So if $d(0)$ is sufficiently small, the line $y=y_{2}(x)$ intersects the curve $y=y_{1}(x)$ at points $x_{1}, x_{2}, \cdots$, with $x_{1}(d(0))<x_{0}<x_{2}(d(0))<\cdots$. Thus the inequality $y_{1}(x) \leq y_{2}(x)$ is valid in disjoint intervals $\left[0, x_{1}(d(0))\right]$ and $\left[x_{2}(d(0)), x_{3}(d(0))\right], \cdots$. Because $d(t)$ is continuous, we have that $d(t)^{1 / 2}<x_{1}(d(0))$ for all $t<\infty$, provided we choose $d(0)^{1 / 2}<x_{0}$. Since $x_{1}(d(0)) \rightarrow 0$ as $d(0) \rightarrow 0$, we deduce the nonlinear stability in terms of the distance functional $d(t)^{1 / 2}$.

\section{Acknowledgements}

This research is supported partly by NSF grants DMS-0603815 and DMS-0505460. We thank the referees for comments and corrections.

\section{REFERENCES}

[1] Antonov, V. A. Remarks on the problem of stability in stellar dynamics. Soviet Astr, AJ., 4, 859-867 (1961).

[2] Antonov, V. A., Solution of the problem of stability of stellar system Emden's density law and the spherical distribution of velocities, Vestnik Leningradskogo Universiteta, Leningrad University, 1962.

[3] Arnold, V. I., Avez, A., Ergodic problems of classical mechanics, W. A. Benjamin, Inc., New York-Amsterdam 1968.

[4] Arnold, V. I., Mathematical methods of classical mechanics, Springer-Verlag, New YorkHeidelberg, 1978.

[5] Barnes, J.; Hut, P.; Goodman, J., Dynamical instabilities in spherical stellar systems, Astrophysical Journal, vol. 300, p. 112-131, 1986.

[6] Bartholomew, P., On the theory of stability of galaxies, Monthly Notices of the Royal Astronomical Society, Vol. 151, p. 333 (1971).

[7] Bertin, Giuseppe, Dynamics of Galaxies, Cambridge University Press, 2000.

[8] Binney, J., Tremaine, S., Galactic Dynamics. Princeton University Press, 1987.

[9] Doremus, J. P.; Baumann, G.; Feix, M. R., Stability of a Self Gravitating System with Phase Space Density Function of Energy and Angular Momentum, Astronomy and Astrophysics, Vol. 29, p. 401 (1973)

[10] Gillon, D.; Cantus, M.; Doremus, J. P.; Baumann, G., Stability of self-gravitating spherical systems in which phase space density is a function of energy and angular momentum, for spherical perturbations, Astronomy and Astrophysics, vol. 50, no. 3, p. 467-470, 1976.

[11] Fridman, A., Polyachenko, V., Physics of Gravitating System Vol I and II, Springer-Verlag, 1984.

[12] Glassey, Robert T., The Cauchy problem in kinetic theory, SIAM, Philadelphia, PA, 1996.

[13] Goodman, Jeremy, An instability test for nonrotating galaxies, Astrophysical Journal, vol. 329, p. 612-617, 1988 .

[14] Guo, Y., Variational method for stable polytropic galaxies, Arch. Rational Mech. Anal., 147, 225-243, 1999. 
[15] Guo, Y., On generalized Antonov stablility criterion for polytropic steady states, Contem. Math., 263, 85-107, 1999.

[16] Guo, Y., Rein, G., Stable steady states in stellar dynamics, Arch. Rational Mech. Anal., 147, no. 3, 225-243, (1999).

[17] Guo, Y., Rein, G., Existence and stability of Camm type steady states in galactic dynamics, Indiana U. Math. J., 48, 1237-1255, 1999.

[18] Guo, Y., Rein, G., Isotropic steady states in stellar dynamics, Commun. Math. Phys., 219, 2001.

[19] Guo, Y., Rein, G., Isotropic steady states in stellar dynamics revisited., Los Alamos Preprint, 2002.

[20] Henon, M., Numerical Experiments on the Stability of Spherical Stellar Systems, Astronomy and Astrophysics, Vol. 24, p. 229 (1973).

[21] Guo, Y., Rein, G., Stability of the King Model and Symmetric Measure-Preserving Perturbations, Preprint.

[22] Kandrup, H.; Signet, J. F.; A simple proof of dynamical stability for a class of spherical clusters. The Astrophys. J. 298, p. 27-33.(1985)

[23] Kandrup, Henry E., A stability criterion for any collisionless stellar equilibrium and some concrete applications thereof, Astrophysical Journal, vol. 370, p. 312-317, 1991.

[24] King, Ivan R., The structure of star clusters. III. Some simple dynamical models, Astronomical Journal, Vol. 71, p. 64 (1966).

[25] Lin, Zhiwu, Instability of periodicBG waves, Math. Res. Letts., 8, 521-534(2001).

[26] Lin, Zhiwu and Strauss, Walter, Linear stability and instability of relativistic Vlasov-Maxwell systems, to appear in Comm. Pure Appl. Math.

[27] Lin, Zhiwu and Strauss, Walter, Nonlinear stability and instability of relativistic VlasovMaxwell systems, to appear in Comm. Pure Appl. Math.

[28] Lin, Zhiwu and Strauss, Walter, A sharp stability criterion for the Vlasov-Maxwell systems, submitted.

[29] Lynden-Bell, D., The Hartree-Fock exchange operator and the stability of galaxies, Monthly Notices of the Royal Astronomical Society, Vol. 144, p.189, 1969.

[30] Lynden-Bell, D. Lectures on stellar dynamics. Galactic dynamics and N-body simulations (Thessaloniki, 1993), 3-31, Lecture Notes in Phys., 433, Springer, Berlin, 1994.

[31] Merritt, David, Elliptical Galaxy Dynamics, The Publications of the Astronomical Society of the Pacific, Volume 111, Issue 756, pp. 129-168.

[32] Palmer, P. L., Stability of collisionless stellar systems: mechanisms for the dynamical structure of galaxies, Kluwer Academic Publishers, 1994.

[33] Perez, Jerome and Aly, Jean-Jacques, Stability of spherical stellar systems - I. Analytical results, Monthly Notices of the Royal Astronomical Society, Volume 280, Issue 3, pp. 689699, 1996.

[34] Pfaffelmoser, K, Global classical solutions of the Vlasov-Poisson system in three dimensions for general initial data, J. Differential Equations 95 (1992), no. 2, 281-303.

[35] Rein, G.: Collisionless Kinetic Equations from Astrophysics - The Vlasov-Poisson system. Preprint 2005.

[36] Schaeffer, Jack, Steady states in galactic dynamics, Arch. Ration. Mech. Anal. 172 (2004), no. $1,1-19$

[37] Sygnet, J. F.; des Forets, G.; Lachieze-Rey, M.; Pellat, R., Stability of gravitational systems and gravothermal catastrophe in astrophysics, Astrophysical Journal, vol. 276, p. 737-745, 1984.

[38] Wan, Y-H., On onlinear stability of isotropic models in stellar dynamics, Arch. Rational. Mech. Anal., 147, (1999) 245-268.

[39] Wolansky, G., On nonlinear stability of polytropic galaxies. Ann. Inst. Henri Poincare. (1999), 16, 15-48.

[40] Yosida, Kôsaku, Functional analysis, Sixth edition. Grundlehren der Mathematischen Wissenschaften, 123. Springer-Verlag, 1980. 
Lefschetz Center for Dynamical Systems, Division of Applied Mathematics, Brown University, Providence, RI 02912, USA

E-mail address: guoy@cfm.brown.edu

Mathematics Department, University of Missouri, Columbia, MO 65211 USA

E-mail address: lin@math.missouri.edu 